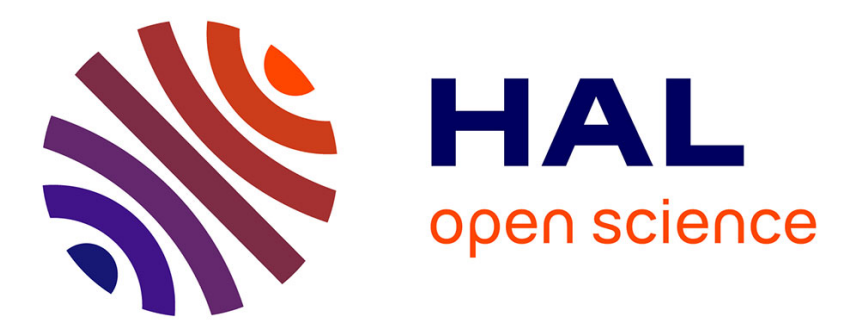

\title{
POD-based model order reduction with an adaptive snapshot selection for a discontinuous Galerkin approximation of the time-domain Maxwell's equations
}

Kun Li, Ting-Zhu Huang, Liang Li, Stéphane Lanteri

\section{- To cite this version:}

Kun Li, Ting-Zhu Huang, Liang Li, Stéphane Lanteri. POD-based model order reduction with an adaptive snapshot selection for a discontinuous Galerkin approximation of the time-domain Maxwell's equations. Journal of Computational Physics, 2019, 396, pp.106-128. 10.1016/j.jcp.2019.05.051 . hal-02433048

\author{
HAL Id: hal-02433048 \\ https://hal.inria.fr/hal-02433048
}

Submitted on 13 Jan 2020

HAL is a multi-disciplinary open access archive for the deposit and dissemination of scientific research documents, whether they are published or not. The documents may come from teaching and research institutions in France or abroad, or from public or private research centers.
L'archive ouverte pluridisciplinaire HAL, est destinée au dépôt et à la diffusion de documents scientifiques de niveau recherche, publiés ou non, émanant des établissements d'enseignement et de recherche français ou étrangers, des laboratoires publics ou privés. 


\title{
POD-based model order reduction with an adaptive snapshot selection for a discontinuous Galerkin approximation of the time-domain Maxwell's equations
}

\author{
Kun $\mathrm{Li}^{\mathrm{a}}$, Ting-Zhu Huang ${ }^{\mathrm{a}, *}$, Liang $\mathrm{Li}^{\mathrm{a}, *}$, Stéphane Lanteri ${ }^{\mathrm{b}}$ \\ ${ }^{a}$ School of Mathematical Sciences, University of Electronic Science and Technology of China, 611731, Chengdu, P.R. \\ China \\ ${ }^{b}$ INRIA, 2004 Route des Lucioles, BP 93, 06902, Sophia Antipolis Cedex, France
}

\begin{abstract}
In this work we report on a reduced-order model (ROM) for the system of time-domain Maxwell's equations discretized by a discontinuous Galerkin (DG) method. We leverage previous results on proper orthogonal decomposition (POD) $[1,2]$, in particular for the wave equation [3], to propose a PODbased ROM with an adaptive snapshot selection strategy where the snapshots are produced by a high order discontinuous Galerkin time-domain (DGTD) solver. The latter is formulated on an unstructured simplicial mesh, and combines a centered scheme for the definition of the numerical fluxes of the electric and magnetic fields at element interfaces with a second order leap-frog $\left(\mathrm{LF}_{2}\right)$ time scheme for the time integration of the associated semi-discrete equations. The POD-based ROM is established by projecting (Galerkin projection) the global semi-discrete DG scheme onto a low-dimensional space generated by the POD basis vectors. Inspired from the approach followed in $[2,3]$, we derive error bounds for the POD-based ROM that is adapted to our particular modeling and discretization settings. The adaptive snapshot selection algorithm exploits the results of this analysis to measure the control error. A snapshot choosing rule aiming at keeping the error estimate close to a target selection error tolerance is proposed, which is similar to the standard rules found in adaptive time-stepping ordinary differential equations (ODEs) solvers. An incremental singular value decomposition (ISVD) algorithm is used to update the SVD on-the-fly when a new snapshot is available. The purpose of this adaptive selection strategy is to save memory without storing snapshots, while producing a smaller error. Numerical experiments for the 2-D time-domain Maxwell's equations nicely illustrate the performance of the resulting POD-based ROM with adaptive snapshot selection.
\end{abstract}

Keywords: time-domain Maxwell's equations, discontinuous Galerkin method, model order reduction, proper orthogonal decomposition, adaptive snapshot selection algorithm

\section{Introduction}

We consider a normalized form of the time-domain Maxwell's equations in a bounded convex domain $\Omega \subset \mathcal{R}^{3}$

$$
\left\{\begin{array}{l}
\mu_{r} \frac{\partial \mathcal{H}}{\partial t}+\operatorname{curl} \mathcal{E}=0, \text { in } \Omega \times\left(0, T_{f}\right], \\
\varepsilon_{r} \frac{\partial \mathcal{E}}{\partial t}-\operatorname{curl} \mathcal{H}=0, \text { in } \Omega \times\left(0, T_{f}\right],
\end{array}\right.
$$

where $\mathcal{E}$ and $\mathcal{H}$ respectively denote the electric and magnetic fields; $T_{f}$ is the final time; $\varepsilon_{r}$ and $\mu_{r}$ are the relative electric permittivity and magnetic permeability parameters. Details on how to obtain the

\footnotetext{
${ }^{*}$ Corresponding authors

Email addresses: rclikun@163.com (Kun Li), tzhuang@uestc.edu.cn (Ting-Zhu Huang), plum_liliang@uestc.edu.cn, plum.liliang@gmail.com (Liang Li), Stephane.Lanteri@inria.fr (Stéphane Lanteri)
} 
normalized form (1) can be found in [4]. The boundary conditions are given by

$$
\left\{\begin{array}{l}
\mathbf{n} \times \mathcal{E}=0, \text { on } \Gamma_{m} \\
\mathcal{L}(\mathcal{E}, \mathcal{H})=\mathcal{L}\left(\mathcal{E}^{\text {inc }}, \mathcal{H}^{\text {inc }}\right), \text { on } \Gamma_{a}
\end{array}\right.
$$

where $\Gamma_{m} \cap \Gamma_{a}=\varnothing, \Gamma_{m} \cup \Gamma_{a}=\partial \Omega$, and $\mathcal{L}(\mathcal{E}, \mathcal{H})=\mathbf{n} \times \mathcal{E}+Z \mathbf{n} \times(\mathbf{n} \times \mathcal{H}) ; \mathbf{n}$ denotes the unit normal vector pointing outward to $\partial \Omega, \mathcal{E}^{\text {inc }}$ and $\mathcal{H}^{\text {inc }}$ are the incident fields, and $Z=\sqrt{\mu_{r} / \varepsilon_{r}}$. The first relation of $(2)$ states a perfect electric conductor (PEC) condition on $\Gamma_{m}$, while the second relation indicates a first order Silver-Müller absorbing boundary condition $(\mathrm{ABC})$ on $\Gamma_{a}$. The initial conditions are $\mathcal{E}(\mathbf{x}, 0)=\mathcal{E}_{0}(\mathbf{x})$, $\mathcal{H}(\mathbf{x}, 0)=\mathcal{H}_{0}(\mathbf{x})$ for $\mathbf{x} \in \Omega \subset \mathcal{R}^{3}$, and where $\mathcal{E}_{0}, \mathcal{H}_{0}$ denote some given functions.

The discontinuous Galerkin time-domain (DGTD) method has emerged in the last 15 years as an appealing strategy for solving the time-domain Maxwell's equations (1) $[5,6]$, in particular because it shares the advantages of the finite element time-domain (FETD) and finite volume time-domain (FVTD) methods. A space of basis and test functions is defined as in the FETD method on one hand, while the equations are satisfied in a sense closer to the FVTD method on the other hand. Moreover, the DGTD method can deal with general (unstructured, possibly non-conforming) [7] meshes and is an ideal candidate for designing $h p$-adaptive solution strategies [8]. Besides, it is easily parallelizable due to its highly local nature. However, the DGTD method is also a demanding discretization method because of the duplication of the degrees of freedom (DoFs) on the boundaries of the elements, which is greater than the number of DoFs used by a conforming FETD method for the same accuracy. Several approaches can be considered to address this issue such as the use of non-conforming meshes [9] and hybridization of the DG formulation method $[10,11,12]$ for time-harmonic problems. The alternative approach that we consider in this work is model order reduction (MOR) $[2,13,14,15]$. MOR method is useful for accelerating simulations in many fields of science and engineering [16, 17, 18, 19, 20, 21, 22]. In particular, MOR method is also widely used in the context of electromagnetics $[19,23,24,25,26,27$, $28,29,30,31,32]$. The overall goal of MOR can be stated as to reduce the computational requirements while maintaining an acceptable level of accuracy.

Different kinds of reduced-order models (ROM) have been developed, such as simplified models and data-fit models [33]. The alternative ROM that we consider in this study is a projection-based model, which proceeds by identifying a reduced subspace that is constructed to retain the essential character of the system input-output map [33]. There are many approaches for constructing the reduced subspace, see [34] for a detailed survey. One of the most studied methods for establishing the ROM is the proper orthogonal decomposition (POD) method [15], also known as Karhunen-Loéve decomposition, principal component analysis, or singular value decomposition, which uses the solutions of high fidelity numerical simulations or experiments at certain time instants, typically called snapshots, to compute a set of POD basis vectors spanning a low-dimensional space. The ROM is then created by projecting (Galerkin projection) the selected snapshots onto a low-dimensional space. The accuracy of the ROM is directly related to the choice of snapshots $[3,20,27,30,35,36]$. In principle, one should store the snapshots at each time step in order to completely characterize the high fidelity simulation. This is not feasible and unnecessary since very little new information will be provided if snapshots are taken close to each other in time [20, 37]. In [35], Luo et al. presented a reduced-order finite volume element (FVE) formulation based on POD method for parabolic problems, and analyzed the error between the POD-based MOR solution and the usual FVE solution under the condition $\ell^{\frac{3}{2}}=O\left(N_{t}\right)$ with $\ell$ being the the number of the snapshots and $N_{t}$ being the number of all time instances. They concluded that it is unnecessary to take total transient solutions at all time instances $t^{(n)}$ as snapshots. In [27], Kowalski and Jin considered a POD-based MOR with three snapshot selection techniques including uniform in time, logarithmic technique with a focus on early time steps and logarithmic technique with a focus on later time steps, for Maxwell's equations to model the performance of a medical device in the human body. They concluded that the optimal snapshot selection scheme depends on the mathematical model under investigation and the parametric structure of that model. Based on this idea, in [20, 37], Siade et al. presented a simple exponential function for a groundwater POD-based MOR since confined aquifers reach steady state in an exponential manner. However, this strategy is difficult to directly apply to other PDE models. Some recent contributions based on an adaptive snapshot selection method for POD-based MOR are presented in $[1,30,38,39]$. For example, in [30], Sato and Igarashi propose a novel method which determines the adequate number of snapshots automatically based on the ratio to characterize the sudden change in the error between the time steps $t^{(n)}$ and $t^{(n+1)}$ for solving Maxwell's equations used to model eddy current 
problems. In [1], the authors study a novel adaptive snapshot selection method in time that reduces off-line training cost according an error control mechanism for a simple first-order dynamical system. The focus of our work is on a generalized first-order dynamical equations obtained after discretization of (1) using the non-dissipative high order DGTD method introduced in [40]. This DGTD method is formulated on an unstructured simplicial mesh, and combines a centered scheme for the definition of the numerical fluxes of the electric and magnetic fields at element interfaces with a second order leap-frog $\left(\mathrm{LF}_{2}\right)$ time scheme for the time integration of the associated semi-discrete equations.

In the adaptive snapshot selection method, a priori error bound between the high fidelity model and the ROM is required. In [15, 41], Kunisch and Volkwein derive a priori error bounds for POD-based methods to solve a class of parabolic partial differential equations (PDEs). Rathinam and Petzold [13] present a priori error estimates of POD-based MOR and also analyze the effects of a small perturbation on the low-dimensional space generated by the POD basis for a first-order dynamical systems. Amsallem and Hermaniuk derive a priori error estimates for Galerkin ROM of the wave equation in [3]. In the present work, inspired from the approach followed in $[2,3,13]$, we derive error bounds for a POD-based ROM designed for a first-order dynamical system resulting from a DG discretization of the time-domain Maxwell's equations. This is achieved at the semi-discrete level, and at the fully discrete level as well when the semi-discrete POD-based ROM is time integrated by the $\mathrm{LF}_{2}$ scheme. The primary objective of our study is to demonstrate the benefits of the proposed POD-based ROM of the system of time-domain Maxwell equations discretized by a high order DGTD method (referred as POD-DGTD in the sequel), with adaptive snapshot selection based on the error analysis. An incremental SVD (ISVD) algorithm, which is the on-the-fly variant of the SVD [42], is employed for the addition of a snapshot to the POD basis. Besides, we adopted a snapshot selection rule aiming at keeping the error estimate close to a snapshot selection error tolerance when choosing the time interval between snapshots in the adaptive algorithm.

As previously noticed, the herein developed theoretical results, albeit being adapted to specific modeling and discretization settings, are very similar to the ones initially derived in [2,3]. From our point view, the main originality of our study lies in the numerical demonstration of the effectiveness of the proposed adaptive POD-DGTD method for the simulation of time-domain electromagnetic wave propagation in homogeneous media and heterogeneous media as well. From this point of view, the combined theoretical and numerical results presented hereafter, contribute to strengthen the aforementioned achievements for the system of time-domain Maxwell equations. Moreover, because the DGTD method is nowadays recognized as a viable alternative to the widespread finite difference time-domain (FDTD) method [43] when dealing with electromagnetic wave interaction with general geometries and complex media, we believe that this work will offer a novel approach for reducing the computational overhead of a classical DG formulation, which is linked to the drawback of a higher number of degrees of freedom as compared to continuous finite element methods.

The rest of the paper is organized as follows. We briefly recall the DGTD method for solving the time-domain Maxwell's equations in Section 2. The POD-based ROM method is established in Section 3. Moreover, a stability analysis of the POD-based ROM method with $\mathrm{LF}_{2}$ time scheme is presented, and error bounds for the ROM are derived in this section. The ISVD and adaptive snapshot selection algorithms are elaborated in Section 4. Numerical results are presented in Section 5 to show the effectiveness of the proposed method for solving 2-D time-domain Maxwell's equations. We draw conclusions in Section 6.

With a few exceptions, we follow the notational conventions used in [28]. Specifically, we use $v$ to denote a scalar quantity, $\mathbf{V}, \mathbf{v}$ or $\mathcal{V}$ a vector, and $\mathbb{A}$ a matrix, $\mathbb{I}_{n}$ for the $n \times n$ identity matrix, and $0_{n \times m}$ for the $n \times m$ zero matrix. We will omit these indices whenever the sizes of $\mathbb{I}$ and 0 are apparent from the context. The set of real numbers is denoted by $\mathcal{R}$. The superscripts ${ }^{T}$ and $\cdot^{\prime}$ respectively denote the transpose and the time derivative.

\section{Discontinuous Galerkin time-domain method}

We consider a partition $\mathcal{T}_{h}$ of $\Omega$ into a set of elements $K_{i}$ of size $h_{i}=\operatorname{diam}\left(K_{i}\right)$ such that $h=\max _{\forall i \in N_{\Omega}} h_{i}$ with $N_{\Omega}$ the set of indices of the mesh elements. We denote by $F_{h}^{I}$ the union of all interior faces of $\mathcal{T}_{h}$, by $F_{h}^{B}$ the union of all boundary faces of $\mathcal{T}_{h}$, and by $F_{h}=F_{h}^{I} \cup F_{h}^{B}$ the union of all faces. For an element $K_{i} \in \mathcal{T}_{h}$, if $K_{j}$ is an adjacent element, $F_{i j}$ is the common face of $K_{i}$ and $K_{j}$, where the index 
$j$ denotes a fictitious element outside the domain when $F_{i j} \in F_{h}^{B}$. We denote by $\mathcal{V}_{i}$ the set of indices of the face-neighbouring elements of the element $K_{i}$, and by $\mathbf{n}_{i j}$ the unit normal vector oriented from $K_{i}$ to $K_{j}$. For a face $F_{i j} \in F_{h}$, we can define the averaging operator $\{\cdot\}$ as

$$
\{\mathbf{v}\}_{F_{i j}}=\frac{1}{2}\left(\mathbf{v}_{i \mid F_{i j}}+\mathbf{v}_{j \mid F_{i j}}\right),
$$

where $\mathbf{v}_{i \mid F_{i j}}$ and $\mathbf{v}_{j \mid F_{i j}}$ respectively stand for the traces of $\mathbf{v}$ on $F_{i j}$ from the interior of $K_{i}$ and $K_{j}$. Note that for any $F_{i j} \in F_{h}^{I},\{\mathbf{v}\}_{F_{i j}}=\{\mathbf{v}\}_{F_{j i}}$. Each $K_{i} \in \mathcal{T}_{h}$ is assumed to be the image, under a smooth bijective mapping, of a fixed reference element $K_{e}=\{(\xi, \eta, \zeta) \mid \xi, \eta, \zeta \geq 0, \xi+\eta+\zeta \leq 1\}$. For each $K_{i} \in \mathcal{T}_{h}$, we denote by $p_{i} \geq 0$ the local interpolation order, and by $\mathbb{P}_{p_{i}}\left(K_{i}\right)$ the space of nodal polynomials of degree at most $p_{i}$ inside the element $K_{i}$. In this study, the interpolation order $p_{i}$ is assumed to be uniform over all the elements, i.e., $\forall i, j \in N_{\Omega}, p_{i}=p_{j}=p$. We introduce the discontinuous finite element space defined by

$$
\mathbf{V}_{h}=\left\{\mathbf{v} \in\left[L^{2}(\Omega)\right]^{3} \mid \mathbf{v}_{\mid K_{i}} \in\left[\mathbb{P}_{p_{i}}\left(K_{i}\right)\right]^{3}, \quad \forall i \in N_{\Omega}\right\},
$$

where $L^{2}(\Omega)$ is the space of square integrable functions in the domain $\Omega$. The functions in $\mathbf{V}_{h}$ are continuous inside each element and discontinuous across the interfaces between elements. For two vectorial functions $\mathbf{u}, \mathbf{v} \in\left[L^{2}(D)\right]^{3},(\mathbf{u}, \mathbf{v})_{D}$ stands for $\int_{D}(\mathbf{u} \cdot \mathbf{v}) d \Omega$, and $\langle\mathbf{u}, \mathbf{v}\rangle_{F}$ stands for $\int_{F}(\mathbf{u} \cdot \mathbf{v}) d l$, where $D$ is a convex domain in $\mathcal{R}^{3}$, and $F$ is an interface in $\mathcal{R}^{3}$.

The DG method seeks an approximate solution $\left(\mathbf{E}_{h}, \mathbf{H}_{h}\right)$ in the space $\mathbf{V}_{h} \times \mathbf{V}_{h}$ such that for all $K_{i}$ in $\mathcal{T}_{h}$

$$
\begin{cases}\left(\mu_{r} \frac{\partial \mathbf{H}_{h}}{\partial t}, \mathbf{v}\right)_{K_{i}}+\left(\operatorname{curl} \mathbf{E}_{h}, \mathbf{v}\right)_{K_{i}}=0, & \forall \mathbf{v} \in \mathbf{V}_{h} \\ \left(\varepsilon_{r} \frac{\partial \mathbf{E}_{h}}{\partial t}, \mathbf{v}\right)_{K_{i}}-\left(\operatorname{curl} \mathbf{H}_{h}, \mathbf{v}\right)_{K_{i}}=0, & \forall \mathbf{v} \in \mathbf{V}_{h}\end{cases}
$$

Integration by partis of (3) and then replacing the boundary terms by numerical traces $\widetilde{\mathbf{E}}_{h}$ and $\widetilde{\mathbf{H}}_{h}$, we have

$$
\left\{\begin{array}{l}
\left(\mu_{r} \frac{\partial \mathbf{H}_{h}}{\partial t}, \mathbf{v}\right)_{K_{i}}+\left(\mathbf{E}_{h}, \operatorname{curl} \mathbf{v}\right)_{K_{i}}-\left\langle\widetilde{\mathbf{E}}_{h} \times \mathbf{n}, \mathbf{v}\right\rangle_{\partial K_{i}}=0, \quad \forall \mathbf{v} \in \mathbf{V}_{h} \\
\left(\varepsilon_{r} \frac{\partial \mathbf{E}_{h}}{\partial t}, \mathbf{v}\right)_{K_{i}}-\left(\mathbf{H}_{h}, \operatorname{curl} \mathbf{v}\right)_{K_{i}}+\left\langle\widetilde{\mathbf{H}}_{h} \times \mathbf{n}, \mathbf{v}\right\rangle_{\partial K_{i}}=0, \quad \forall \mathbf{v} \in \mathbf{V}_{h}
\end{array}\right.
$$

Proper choice of numerical traces $\widetilde{\mathbf{E}}_{h}$ and $\widetilde{\mathbf{H}}_{h}$ is essential for the correctness and the convergence of the DG scheme. For the sake of simplicity, we make use in this study of the centered numerical traces, i.e.,

$$
\widetilde{\mathbf{E}}_{h}=\left\{\mathbf{E}_{h}\right\}_{F_{i j}}, \quad \widetilde{\mathbf{H}}_{h}=\left\{\mathbf{H}_{h}\right\}_{F_{i j}}, \quad \forall i \in N_{\Omega}, \forall j \in \mathcal{V}_{i} .
$$

which is the option adopted and studied in [40]. We denote by $\left(\mathbf{E}_{i}, \mathbf{H}_{i}\right)$ the approximations $\left(\mathbf{E}_{h \mid K_{i}}, \mathbf{H}_{h \mid K_{i}}\right)$ restricted to the element $K_{i}$. As in a classical finite element setting, the fields $\mathbf{E}_{i}$ and $\mathbf{H}_{i}$ are linear combinations of the basis vectors on the element $K_{i}$ with coefficient vectors $\underline{\mathbf{E}}_{i}$ and $\underline{\mathbf{H}}_{i}$, respectively. For each element $K_{i} \in \mathcal{T}_{h}$, a set of scalar basis functions $\left(\varphi_{i k}\right)_{1 \leq k \leq d_{i}}$ is defined, where $d_{i}$ denotes the number of DoFs inside $K_{i}$ for each spatial dimension. For a $3-\mathrm{D}$ problem, we have $3 d_{i}$ DoFs per element for each field, and the three vectorial basis functions are defined as

$$
\Phi_{i k}^{1}=\left(\varphi_{i k}, 0,0\right)^{T}, \Phi_{i k}^{2}=\left(0, \varphi_{i k}, 0\right)^{T}, \Phi_{i k}^{3}=\left(0,0, \varphi_{i k}\right)^{T}, \quad \forall i \in N_{\Omega}, 1 \leq k \leq d_{i} .
$$

$\mathbf{E}_{i}$ is then locally expanded as

$$
\mathbf{E}_{i}=\sum_{m=1}^{3} \sum_{k=1}^{d_{i}} E_{i k}^{m} \Phi_{i k}^{m}=\underline{\mathbf{E}}_{i}^{T} \Phi_{i}
$$

where $\underline{\mathbf{E}}_{i}=\left[\begin{array}{c}\left(E_{i k}^{1}\right)_{1 \leq k \leq d_{i}} \\ \left(E_{i k}^{2}\right)_{1 \leq k \leq d_{i}} \\ \left(E_{i k}^{3}\right)_{1 \leq k \leq d_{i}}\end{array}\right]$ and $\Phi_{i}=\left[\Phi_{i 1}^{1}, \cdots, \Phi_{i d_{i}}^{1}, \Phi_{i 1}^{2}, \cdots, \Phi_{i d_{i}}^{2}, \Phi_{i 1}^{3}, \cdots, \Phi_{i d_{i}}^{3}\right]^{T}$. The definitions for

$\mathbf{H}_{i}$ are similar to those for $\mathbf{E}_{i}$. One can obtain the following semi-discrete formulation in $K_{i}$

$$
\left\{\begin{array}{l}
\mu_{r} \mathbb{M}_{i} \frac{\partial \underline{\mathbf{H}}_{i}}{\partial t}=-\mathbb{K}_{i} \underline{\mathbf{E}}_{i}+\sum_{l \in \mathcal{V}_{i}} \mathbb{S}_{i l} \underline{\mathbf{E}}_{l} \\
\varepsilon_{r} \mathbb{M}_{i} \frac{\partial \underline{\mathbf{E}}_{i}}{\partial t}=\mathbb{K}_{i} \underline{\mathbf{H}}_{i}-\sum_{l \in \mathcal{V}_{i}} \mathbb{S}_{i l} \underline{\mathbf{H}}_{l}
\end{array}\right.
$$


where the $3 d_{i} \times 3 d_{i}$ block diagonal mass matrix $\mathbb{M}_{i}=\operatorname{diag}\left(\widetilde{\mathbb{M}}_{i}^{1}, \widetilde{\mathbb{M}}_{i}^{2}, \widetilde{\mathbb{M}}_{i}^{3}\right)$, the $3 d_{i} \times 3 d_{i}$ block diagonal stiffness matrix $\mathbb{K}_{i}=\operatorname{diag}\left(\widetilde{\mathbb{K}}_{i}^{1}, \widetilde{\mathbb{K}}_{i}^{2}, \widetilde{\mathbb{K}}_{i}^{3}\right)$, and the $3 d_{i} \times 3 d_{l}$ block diagonal surface matrix $\mathbb{S}_{i l}=\operatorname{diag}\left(\widetilde{\mathbb{S}}_{i l}^{1}, \widetilde{\mathbb{S}}_{i l}^{2}, \widetilde{\mathbb{S}}_{i l}^{3}\right)$ are defined by their respective diagonal blocks

$$
\left\{\begin{array}{l}
\left(\widetilde{\mathbb{M}}_{i}^{m}\right)_{j k}=\left(\Phi_{i j}^{m}, \Phi_{i k}^{m}\right)_{K_{i}}, \\
\left(\widetilde{\mathbb{K}}_{i}^{m}\right)_{j k}=\frac{1}{2}\left[\left(\Phi_{i j}^{m}, \operatorname{curl} \Phi_{i k}^{m}\right)_{K_{i}}+\left(\operatorname{curl} \Phi_{i j}^{m}, \Phi_{i k}^{m}\right)_{K_{i}}\right], \\
\left(\widetilde{\mathbb{S}}_{i l}^{m}\right)_{j k}=\frac{1}{2}\left\langle\Phi_{i j}^{m}, \Phi_{l k}^{m} \times \mathbf{n}_{i l}\right\rangle_{F_{i l}},
\end{array}\right.
$$

where the dimension of $\widetilde{\mathbb{M}}_{i}^{m}, \widetilde{\mathbb{K}}_{i}^{m}$, and $\widetilde{\mathbb{S}}_{i l}^{m}$ are $d_{i} \times d_{i}, d_{i} \times d_{i}$, and $d_{i} \times d_{l}$, respectively, $m=\{1,2,3\}$, and $d_{l}$ denotes the number of DoFs on the face $F_{i l} \in \mathcal{V}_{i}$. Concerning the time discretization, we adopt the $\mathrm{LF}_{2}$ scheme. The combination of the centered numerical traces in the DG formulation with the $\mathrm{LF}_{2}$ scheme for time integration of (6) leads to a non-dissipative DGTD method [40]. We divide the time interval $\left[0, T_{f}\right]$ into $N_{t}$ equally spaced subintervals as

$$
0=t^{(0)}<t^{(1)}<\cdots<t^{\left(N_{t}\right)}=T_{f},
$$

where $t^{(n)}=n \Delta t\left(n \in\left\{0,1, \cdots, N_{t}\right\}\right)$ and $\Delta t$ denotes the time step size, which is constrained to a Courant-Friedrichs-Lewy (CFL) condition [40]. Then, the fully discrete scheme is given by

$$
\left\{\begin{array}{l}
\varepsilon_{r} \mathbb{M}_{i} \frac{\underline{\mathbf{E}}_{i}^{(n+1)}-\underline{\mathbf{E}}_{i}^{(n)}}{\Delta t}=\mathbb{K}_{i} \underline{\mathbf{H}}_{i}^{\left(n+\frac{1}{2}\right)}-\sum_{l \in \nu_{i}} \mathbb{S}_{i l} \underline{\mathbf{H}}_{l}^{\left(n+\frac{1}{2}\right)}, \\
\mu_{r} \mathbb{M}_{i} \frac{\underline{\mathbf{H}}_{i}^{\left(n+\frac{3}{2}\right)}-\underline{\mathbf{H}}_{i}^{\left(n+\frac{1}{2}\right)}}{\Delta t}=-\mathbb{K}_{i} \underline{\mathbf{E}}_{i}^{(n+1)}+\sum_{l \in \nu_{i}} \mathbb{S}_{i l} \underline{\mathbf{E}}_{l}^{(n+1)},
\end{array} \quad n=0,1, \cdots, N_{t}-1 .\right.
$$

In order to construct the POD-based ROM, we need a global formulation of the problem that we state below for the semi-discrete system (5). By gathering the electric and magnetic DoFs in each element into column vectors of size $N=3 \sum_{i \in N_{\Omega}} d_{i}$, denoted by $\underline{\mathbf{E}}_{h}$ and $\underline{\mathbf{H}}_{h}$ respectively, the local semi-discrete system (5) for each element $K_{i}$ of the mesh can be transformed into the following global semi-discrete system

$$
\left\{\begin{array}{l}
\mu_{r} \mathbb{M} \frac{\partial \underline{\mathbf{H}}_{h}}{\partial t}=-\mathbb{K} \underline{\mathbf{E}}_{h}+\mathbb{S}^{i} \underline{\mathbf{E}}_{h}+\mathbb{S}^{e} \underline{\hat{\mathbf{E}}}_{h}+\mathbf{B}^{e}(t) \\
\varepsilon_{r} \mathbb{M} \frac{\partial \underline{\mathbf{E}_{h}}}{\partial t}=\mathbb{K} \underline{\mathbf{H}}_{h}-\mathbb{S}^{i} \underline{\mathbf{H}}_{h}-\mathbb{S}^{h} \underline{\widehat{\mathbf{H}}}_{h}-\mathbf{B}^{h}(t)
\end{array}\right.
$$

where $\mathbb{M}$ and $\mathbb{K}$ are $N \times N$ block diagonal matrices, whose diagonal blocks are equal to $\mathbb{M}_{i}$ and $\mathbb{K}_{i}$, respectively. $\mathbb{S}^{i}$ is an $N \times N$ block sparse matrix whose non-zero blocks are equal to $\mathbb{S}_{i l}$. $\mathbb{S}^{h}$ and $\mathbb{S}^{e}$ are also $N \times N$ block diagonal matrices whose non-zero blocks are respectively equal to $\mathbb{S}_{i l}$ and $-\mathbb{S}_{i l}$ if $F_{i l} \in F_{h}^{B} \cap \Gamma_{m}$, assuming that $\widehat{\mathbf{E}}_{h}=\underline{\mathbf{E}}_{h}$ and $\underline{\hat{\mathbf{H}}}_{h}=\underline{\mathbf{H}}_{h}$ on a face of $\Gamma_{m}$ from (2). The non-zero blocks of $\mathbb{S}^{h}$ and $\mathbb{S}^{e}$ are respectively equal to $\mathbb{S}_{i l}^{h}$ and $\mathbb{S}_{i l}^{e}$ if $F_{i l} \in F_{h}^{B} \cap \Gamma_{a}$, where the $3 d_{i} \times 3 d_{l}$ block diagonal surface matrix $\mathbb{S}_{i l}^{h}=\operatorname{diag}\left(\widetilde{\mathbb{S}}_{i l}^{h, 1}, \widetilde{\mathbb{S}}_{i l}^{h, 2}, \widetilde{\mathbb{S}}_{i l}^{h, 3}\right)$ and $\mathbb{S}_{i l}^{e}=\operatorname{diag}\left(\widetilde{\mathbb{S}}_{i l}^{e, 1}, \widetilde{\mathbb{S}}_{i l}^{e, 2}, \widetilde{\mathbb{S}}_{i l}^{e, 3}\right)$ are defined by their respective diagonal blocks

$$
\left\{\begin{array}{l}
\left(\widetilde{\mathbb{S}}_{i l}^{h, m}\right)_{j k}=-\frac{1}{2} Z_{i}^{-1}\left\langle\Phi_{i j}^{m},\left(\Phi_{l k}^{m} \times \mathbf{n}_{i l}\right) \times \mathbf{n}_{i l}\right\rangle_{F_{i l}}, \quad 1 \leq m \leq 3 . \\
\left(\widetilde{\mathbb{S}}_{i l}^{e, m}\right)_{j k}=\frac{1}{2} Z_{i}\left\langle\Phi_{i j}^{m},\left(\Phi_{l k}^{m} \times \mathbf{n}_{i l}\right) \times \mathbf{n}_{i l}\right\rangle_{F_{i l}},
\end{array}\right.
$$

And one can find that $\widehat{\underline{\mathbf{E}}}_{h}=\underline{\mathbf{H}}_{h}$ and $\underline{\widehat{\mathbf{H}}}_{h}=\underline{\mathbf{E}}_{h}$ on a face on $\Gamma_{a}$ from $(2)$. $\mathbf{B}^{e}(t)=\mathbb{S}^{\text {inc }} \mathbf{E}^{\text {inc }}-\mathbb{S}^{e} \mathbf{H}^{\text {inc }}$ and $\mathbf{B}^{h}(t)=\mathbb{S}^{\text {inc }} \mathbf{H}^{\text {inc }}-\mathbb{S}^{h} \mathbf{E}^{\text {inc }}$ are $N \times 1$ time-dependent vectors when $F_{i l} \in F_{h}^{B} \cap \Gamma_{a}$, where $\mathbb{S}^{\text {inc }}$ is an $N \times N$ block sparse matrix whose non-zero blocks are equal to $\mathbb{S}_{i l} ; \mathbf{B}^{h}(t)$. $\mathbf{B}^{e}(t)$ are $N \times 1$ zero vectors when $F_{i l} \in F_{h}^{B} \cap \Gamma_{m}$. In particular, $\mathbb{M}$ is a symmetric positive definite matrix, $\mathbb{K}$ and $\mathbb{S}^{i}$ are symmetric matrices, and $\mathbb{S}^{e}$ and $\mathbb{S}^{h}$ are skew-symmetric matrices. Besides, $\mathbb{S}^{e}=-\mathbb{S}^{h}$ when $\Gamma_{a}=\varnothing$. For detailed descriptions of these matrices, see [28].

\section{POD-based model order reduction and error estimates}

In this section, we present the formulations of the POD-based ROM, and analyze the stability and the error bounds based on appropriate norms to measure the ROM error at both the semi-discrete and fully discrete levels. 


\subsection{Reduced-order model}

We equidistantly or unevenly extract $\ell$ solutions $\mathbf{u}_{h}^{n_{i}}\left(0 \leq n_{1} \leq \cdots \leq n_{\ell} \leq N_{t}, \ell \ll N_{t}\right)$ from the transient solutions $\left\{\mathbf{u}_{h}^{i}\right\}_{i=0}^{N_{t}}\left(\mathbf{u}_{h}^{i}=\underline{\mathbf{E}}_{h}^{(i)}, \underline{\mathbf{H}}_{h}^{\left(i+\frac{1}{2}\right)}\right)$ of the DGTD method (7) as snapshot vectors. Then, we formulate two $N \times \ell$ snapshot matrices $\mathbb{A}_{\mathbf{E}}$ and $\mathbb{A}_{\mathbf{H}}$ as follows

$$
\mathbb{A}_{\mathbf{u}}=\left[\begin{array}{cccc}
\mathbf{u}_{h, 1}^{n_{1}} & \mathbf{u}_{h,}^{n_{2}} & \cdots & \mathbf{u}_{h, 1}^{n_{\ell}} \\
\mathbf{u}_{h, 2}^{n_{1}} & \mathbf{u}_{h, 2}^{n_{2}} & \cdots & \mathbf{u}_{h, 2}^{n_{\ell}} \\
\vdots & \vdots & \ddots & \vdots \\
\mathbf{u}_{h, N}^{n_{1}} & \mathbf{u}_{h, N}^{n_{2}} & \cdots & \mathbf{u}_{h, N}^{n_{\ell}}
\end{array}\right], \quad \mathbf{u}=\mathbf{E}, \mathbf{H} .
$$

Let

$$
\mathbb{A}_{\mathbf{u}}=\mathbb{U}_{\mathbf{u}}\left[\begin{array}{cc}
\sum_{r_{\mathbf{u}} \times r_{\mathbf{u}}}^{\mathbf{u}} & 0_{r_{\mathbf{u}} \times\left(\ell-r_{\mathbf{u}}\right)} \\
0_{\left(N-r_{\mathbf{u}}\right) \times r_{\mathbf{u}}} & 0_{\left(N-r_{\mathbf{u}}\right) \times\left(\ell-r_{\mathbf{u}}\right)}
\end{array}\right] \mathbb{V}_{\mathbf{u}}^{T}, \quad \mathbf{u}=\mathbf{E}, \mathbf{H},
$$

be the SVD of $\mathbb{A}_{\mathbf{u}}$, where $\mathbb{U}_{\mathbf{u}}$ and $\mathbb{V}_{\mathbf{u}}$ are $N \times N$ and $\ell \times \ell$ unitary matrices, $r_{\mathbf{u}}$ is the rank of $\mathbb{A}_{\mathbf{u}}$, and

$$
\Sigma_{r_{\mathbf{u}} \times r_{\mathbf{u}}}^{\mathbf{u}}=\operatorname{diag}\left(\sigma_{\mathbf{u}, 1}, \sigma_{\mathbf{u}, 2}, \cdots, \sigma_{\mathbf{u}, r_{\mathbf{u}}}\right),
$$

with $\sigma_{\mathbf{u}, 1} \geq \sigma_{\mathbf{u}, 2} \geq \cdots \geq \sigma_{\mathbf{u}, r_{\mathbf{u}}} \geq 0$ being the singular values of $\mathbb{A}_{\mathbf{u}}$. Given a number $d_{\mathbf{u}}(\mathbf{u}=\mathbf{E}, \mathbf{H})$, the POD method defines a basis $\Psi_{\mathbf{u}}$ created by the left singular vectors corresponding to the $d_{\mathbf{u}}$ greatest singular values of $\mathbb{A}_{\mathbf{u}}$. The reduced-order approximation of the fields takes the form based on a Galerkin ansatz

$$
\underline{\mathbf{E}}_{h} \approx \underline{\mathbf{E}}_{h}^{r}=\Psi_{\mathbf{E}} \alpha_{\mathbf{E}}(t), \underline{\mathbf{H}}_{h} \approx \underline{\mathbf{H}}_{h}^{r}=\Psi_{\mathbf{H}} \alpha_{\mathbf{H}}(t),
$$

where $\alpha_{\mathbf{u}}(t) \in \mathcal{R}^{d_{\mathbf{u}}}(\mathbf{u}=\mathbf{E}, \mathbf{H})$ is the state vector of the ROM, and $\underline{\mathbf{E}}_{h}^{r}, \underline{\mathbf{H}}_{h}^{r}$ denote the reduced-order solutions. We obtain the residual vectors by substituting (10) into (8)

$$
\left\{\begin{array}{l}
\operatorname{res}_{\mathbf{H}}(t)=\mu_{r} \mathbb{M} \Psi_{\mathbf{H}} \alpha_{\mathbf{H}}^{\prime}(t)+\left(\mathbb{K}-\mathbb{S}^{i}\right) \Psi_{\mathbf{E}} \alpha_{\mathbf{E}}(t)-\mathbb{S}^{e} \widehat{\Psi}_{\mathbf{E}} \widehat{\alpha}_{\mathbf{E}}(t)-\mathbf{B}^{e}(t), \\
\operatorname{res}_{\mathbf{E}}(t)=\varepsilon_{r} \mathbb{M} \Psi_{\mathbf{E}} \alpha_{\mathbf{E}}^{\prime}(t)-\left(\mathbb{K}-\mathbb{S}^{i}\right) \Psi_{\mathbf{H}} \alpha_{\mathbf{H}}(t)+\mathbb{S}^{h} \widehat{\Psi}_{\mathbf{H}} \widehat{\alpha}_{\mathbf{H}}(t)+\mathbf{B}^{h}(t),
\end{array}\right.
$$

with

and

$$
\widehat{\alpha}_{\mathbf{E}}(t)=\left\{\begin{array}{ll}
\alpha_{\mathbf{E}}(t), & \text { if } F_{h}^{B}=\Gamma_{m}, \\
\alpha_{\mathbf{H}}(t), & \text { if } F_{h}^{B}=\Gamma_{a},
\end{array} \quad \widehat{\alpha}_{\mathbf{H}}(t)=\left\{\begin{array}{lll}
\alpha_{\mathbf{H}}(t), & \text { if } F_{h}^{B}=\Gamma_{m}, \\
\alpha_{\mathbf{E}}(t), & \text { if } F_{h}^{B}=\Gamma_{a},
\end{array}\right.\right.
$$

$$
\widehat{\Psi}_{\mathbf{E}}=\left\{\begin{array}{ll}
\Psi_{\mathbf{E}}, & \text { if } F_{h}^{B}=\Gamma_{m}, \\
\Psi_{\mathbf{H}}, & \text { if } F_{h}^{B}=\Gamma_{a},
\end{array} \widehat{\Psi}_{\mathbf{H}}= \begin{cases}\Psi_{\mathbf{H}}, & \text { if } F_{h}^{B}=\Gamma_{m}, \\
\Psi_{\mathbf{E}}, & \text { if } F_{h}^{B}=\Gamma_{a} .\end{cases}\right.
$$

The residual vectors $\operatorname{res}_{\mathbf{u}}(t)(\mathbf{u}=\mathbf{E}, \mathbf{H})$ are required to be orthogonal to the reduced-order space, which is spanned by the reduced-order basis $\Psi_{\mathbf{u}}$, by applying the Galerkin projection

$$
\Psi_{\mathbf{H}}^{T} r e s_{\mathbf{H}}(t) \equiv 0, \quad \Psi_{\mathbf{E}}^{T} r e s_{\mathbf{E}}(t) \equiv 0 .
$$

The global semi-discrete ROM system is then written as

$$
\left\{\begin{array}{l}
\mu_{r} \Psi_{\mathbf{H}}^{T} \mathbb{M} \Psi_{\mathbf{H}} \alpha_{\mathbf{H}}^{\prime}(t)=\Psi_{\mathbf{H}}^{T}\left(-\mathbb{K}+\mathbb{S}^{i}\right) \Psi_{\mathbf{E}} \alpha_{\mathbf{E}}(t)+\Psi_{\mathbf{H}}^{T} \mathbb{S}^{e} \widehat{\Psi}_{\mathbf{E}} \widehat{\alpha}_{\mathbf{E}}(t)+\Psi_{\mathbf{H}}^{T} \mathbf{B}^{e}(t), \\
\varepsilon_{r} \Psi_{\mathbf{E}}^{T} \mathbb{M} \Psi_{\mathbf{E}} \alpha_{\mathbf{E}}^{\prime}(t)=\Psi_{\mathbf{E}}^{T}\left(\mathbb{K}-\mathbb{S}^{i}\right) \Psi_{\mathbf{H}} \alpha_{\mathbf{H}}(t)-\Psi_{\mathbf{E}}^{T} \mathbb{S}^{h} \widehat{\Psi}_{\mathbf{H}} \widehat{\alpha}_{\mathbf{H}}(t)-\Psi_{\mathbf{E}}^{T} \mathbf{B}^{h}(t),
\end{array}\right.
$$

with the initial conditions

$$
\left\{\begin{array}{l}
\alpha_{\mathbf{E}}(0)=\left(\Psi_{\mathbf{E}}^{T} \mathbb{M} \Psi_{\mathbf{E}}\right)^{-1} \Psi_{\mathbf{E}}^{T} \mathbb{M} \underline{\mathbf{E}}_{h}(0), \\
\alpha_{\mathbf{H}}(0)=\left(\Psi_{\mathbf{H}}^{T} \mathbb{M} \Psi_{\mathbf{H}}\right)^{-1} \Psi_{\mathbf{H}}^{T} \mathbb{M} \underline{\mathbf{H}}_{h}(0) .
\end{array}\right.
$$

The local fully discrete system (7) for each element $K_{i}$ can be transformed into a global system

$$
\left\{\begin{array}{l}
\varepsilon_{r} \mathbb{M} \frac{\underline{\mathbf{E}}_{h}^{(n+1)}-\underline{\mathbf{E}}_{h}^{(n)}}{\Delta t}=\left(\mathbb{K}-\mathbb{S}^{i}\right) \underline{\mathbf{H}}_{h}^{\left(n+\frac{1}{2}\right)}-\mathbb{S}^{h} \underline{\widehat{\mathbf{H}}}_{h}^{\left(n+\frac{1}{2}\right)}-\mathbf{B}^{h}(n \Delta t), \\
\mu_{r} \mathbb{M} \frac{\underline{\mathbf{H}}_{h}^{\left(n+\frac{3}{2}\right)}-\underline{\mathbf{H}}_{h}^{\left(n+\frac{1}{2}\right)}}{\Delta t}=\left(-\mathbb{K}+\mathbb{S}^{i}\right) \underline{\mathbf{E}}_{h}^{(n+1)}+\mathbb{S}^{e} \underline{\widehat{\mathbf{E}}}_{h}^{(n+1)}+\mathbf{B}^{e}\left(\left(n+\frac{1}{2}\right) \Delta t\right) .
\end{array}\right.
$$


By applying the approximation

$$
\left\{\begin{array}{l}
\underline{\mathbf{E}}_{h}^{(n)} \approx \underline{\mathbf{E}}_{h}^{r,(n)}=\Psi_{\mathbf{E}} \alpha_{\mathbf{E}}^{(n)}, \\
\underline{\mathbf{H}}_{h}^{\left(n+\frac{1}{2}\right)} \approx \underline{\mathbf{H}}_{h}^{r,\left(n+\frac{1}{2}\right)}=\Psi_{\mathbf{H}} \alpha_{\mathbf{H}}^{\left(n+\frac{1}{2}\right)},
\end{array}\right.
$$

we obtain the fully discrete reduced-order model, for $n=0,1, \cdots, N_{t}-1$

$$
\left\{\begin{aligned}
\varepsilon_{r} \Psi_{\mathbf{E}}^{T} \mathbb{M} \Psi_{\mathbf{E}} \frac{\alpha_{\mathbf{E}}^{(n+1)}-\alpha_{\mathbf{E}}^{(n)}}{\Delta t} & =\Psi_{\mathbf{E}}^{T}\left(\mathbb{K}-\mathbb{S}^{i}\right) \Psi_{\mathbf{H}} \alpha_{\mathbf{H}}^{\left(n+\frac{1}{2}\right)}-\Psi_{\mathbf{E}}^{T} \mathbb{S}^{h} \widehat{\Psi}_{\mathbf{H}} \widehat{\alpha}_{\mathbf{H}}^{\left(n+\frac{1}{2}\right)} \\
& -\Psi_{\mathbf{E}}^{T} \mathbf{B}^{h}(n \Delta t), \\
\mu_{r} \Psi_{\mathbf{H}}^{T} \mathbb{M} \Psi_{\mathbf{H}} \frac{\alpha_{\mathbf{H}}^{\left(n+\frac{3}{2}\right)}-\alpha_{\mathbf{H}}^{\left(n+\frac{1}{2}\right)}}{\Delta t} & =\Psi_{\mathbf{H}}^{T}\left(-\mathbb{K}+\mathbb{S}^{i}\right) \Psi_{\mathbf{E}} \alpha_{\mathbf{E}}^{(n+1)}+\Psi_{\mathbf{H}}^{T} \mathbb{S}^{e} \widehat{\Psi}_{\mathbf{E}} \widehat{\alpha}_{\mathbf{E}}^{(n+1)} \\
& +\Psi_{\mathbf{H}}^{T} \mathbf{B}^{e}\left(\left(n+\frac{1}{2}\right) \Delta t\right),
\end{aligned}\right.
$$

with the initial conditions

$$
\left\{\begin{array}{l}
\alpha_{\mathbf{E}}^{(0)}=\left(\Psi_{\mathbf{E}}^{T} \mathbb{M} \Psi_{\mathbf{E}}\right)^{-1} \Psi_{\mathbf{E}}^{T} \mathbb{M} \mathbf{E}_{h}^{(0)} \\
\alpha_{\mathbf{H}}^{\left(\frac{1}{2}\right)}=\left(\Psi_{\mathbf{H}}^{T} \mathbb{M} \Psi_{\mathbf{H}}\right)^{-1} \Psi_{\mathbf{H}}^{T} \mathbb{M} \mathbf{H}_{h}^{\left(\frac{1}{2}\right)}
\end{array}\right.
$$

Reamrk 1. The matrices $\Psi_{\mathbf{E}}^{T} \mathbb{M} \Psi_{\mathbf{E}}$ and $\Psi_{\mathbf{H}}^{T} \mathbb{M} \Psi_{\mathbf{H}}$ in (13) and in (17) are $d_{\mathbf{E}} \times d_{\mathbf{E}}$ and $d_{\mathbf{H}} \times d_{\mathbf{H}}$ respectively, thus of small size. So their inversion is not computationally expensive, and the POD-DGTD method is still effective even if theses matrices are dense. The inverse of the matrix $\Psi_{\mathbf{u}}^{T} \mathbb{M} \Psi_{\mathbf{u}}(\mathbf{u}=\mathbf{E}, \mathbf{H})$ exists because $\mathbb{M}$ is a real symmetric positive definite matrix and $\Psi_{\mathbf{u}}$ consists of the left singular vectors corresponding to the $d_{\mathbf{u}}$ greatest singular values of $\mathbb{A}_{\mathbf{u}}$. In particular, $\Psi_{\mathbf{u}}^{T} \mathbb{M} \Psi_{\mathbf{u}}$ is also a symmetric positive definite matrix.

Reamrk 2. The general goal of the proposed POD-DGTD method is to reduce the complexity of a full time-domain simulation in order to address problems such as design optimization or uncertainty quantification that will require performing many simulations. A specific goal is to study the applicability of POD for reducing the complexity of the full time-domain simulation and running with the same basis for different configurations. The snapshot vectors in this work are chosen from the full DGTD solutions. However, one may obtain the ensemble of snapshots from physical system trajectories by drawing samples from experiments and interpolation (or data assimilation). For example, we can run the full DGTD simulation with a reference parameter, and construct the snapshots and the POD basis. Then we can run the POD-DGTD simulations when the parameter changes at a much lower cost without running the full DGTD simulation, as we show in the numerical test for the scattering of a plane wave by a dielectric disk, see Figure \%.

\subsection{Stability analysis}

The resulting full DGTD method with $\mathrm{LF}_{2}$ time scheme (14) is analyzed in [44] where it is shown that the method is non-dissipative, conserves a discrete form of the electromagnetic energy and is stable under the CFL condition

$$
\Delta t<\frac{2}{d_{N}}, \text { with } d_{N}=\left\|\left(\mathbb{M}^{\mu_{r}}\right)^{-\frac{1}{2}}\left(\mathbb{K}-\mathbb{S}^{i}-\mathbb{S}^{e}\right)\left(\mathbb{M}^{\varepsilon_{r}}\right)^{-\frac{1}{2}}\right\|
$$

where the matrix $\mathbb{M}^{\sigma}=\sigma \mathbb{M}\left(\sigma\right.$ stands for $\varepsilon_{r}$ or $\left.\mu_{r}\right),\|\cdot\|$ denotes a canonical norm of a matrix $(\forall \mathbf{x},\|\mathbb{A} \mathbf{x}\| \leq$ $\|\mathbb{A}\|\|\mathbf{x}\|)$, and $(\cdot)^{-\frac{1}{2}}$ is the inverse square root of a matrix. The stability of the POD-DGTD scheme with the $\mathrm{LF}_{2}$ time scheme is now analyzed by using an energy method, where a quadratic form plays the role of a Lyapunov function of the whole set of numerical unknowns.

Definition 1. We consider the following discrete electromagnetic energy in the whole domain $\Omega$

$$
\Theta^{r, n}=\frac{1}{2}\left[\left(\underline{\mathbf{E}}_{h}^{r,(n)}\right)^{T} \mathbb{M}^{\varepsilon_{r}} \underline{\mathbf{E}}_{h}^{r,(n)}+\left(\underline{\mathbf{H}}_{h}^{r,\left(n+\frac{1}{2}\right)}\right)^{T} \mathbb{M}^{\mu_{r}} \underline{\mathbf{H}}_{h}^{r,\left(n-\frac{1}{2}\right)}\right] .
$$


In the following, we shall verify that $\Theta^{r, n}$ is exactly conserved in the absence of the first order Silver-Müller ABC, i.e., $\Delta \Theta^{r}=\Theta^{r, n+1}-\Theta^{r, n}=0$.

Theorem 1. Using the POD-DGTD scheme (15)-(16) with PEC condition only, the global discrete energy (18) is exactly conserved.

Proof. Based on the definition of the electromagnetic energy, we have

$$
\begin{aligned}
\Theta^{r, n+1}-\Theta^{r, n} & =\frac{1}{2}\left(\alpha_{\mathbf{E}}^{(n+1)}+\alpha_{\mathbf{E}}^{(n)}\right)^{T}\left(\Psi_{\mathbf{E}}^{T} \mathbb{M}^{\varepsilon_{r}} \Psi_{\mathbf{E}}\right)\left(\alpha_{\mathbf{E}}^{(n+1)}-\alpha_{\mathbf{E}}^{(n)}\right) \\
& +\frac{1}{2} \alpha_{\mathbf{H}}^{\left(n+\frac{1}{2}\right)}\left(\Psi_{\mathbf{H}}^{T} \mathbb{M}^{\mu_{r}} \Psi_{\mathbf{H}}\right)\left(\alpha_{\mathbf{H}}^{\left(n+\frac{3}{2}\right)}-\alpha_{\mathbf{H}}^{\left(n-\frac{1}{2}\right)}\right) .
\end{aligned}
$$

We denote by $\alpha_{\mathbf{E}}^{\left[n+\frac{1}{2}\right]}=\frac{\left(\alpha_{\mathbf{E}}^{(n+1)}+\alpha_{\mathbf{E}}^{(n)}\right)}{2}$, and then we have

$$
\begin{aligned}
\Theta^{r, n+1}-\Theta^{r, n} & =\Delta t\left(\alpha_{\mathbf{E}}^{\left[n+\frac{1}{2}\right]}\right)^{T} \Psi_{\mathbf{E}}^{T}\left(\mathbb{K}-\mathbb{S}^{i}-\mathbb{S}^{h}\right) \Psi_{\mathbf{H}} \alpha_{\mathbf{H}}^{\left(n+\frac{1}{2}\right)} \\
& +\Delta t\left(\alpha_{\mathbf{H}}^{\left(n+\frac{1}{2}\right)}\right)^{T} \Psi_{\mathbf{H}}^{T}\left(-\mathbb{K}+\mathbb{S}^{i}+\mathbb{S}^{e}\right) \Psi_{\mathbf{E}} \alpha_{\mathbf{E}}^{\left[n+\frac{1}{2}\right]}
\end{aligned}
$$

Based on the definition of (8), we have

$$
\mathbb{K}=\mathbb{K}^{T}, \mathbb{S}^{i}=\left(\mathbb{S}^{i}\right)^{T}, \mathbb{S}^{h}=-\mathbb{S}^{e}, \text { and }\left(\mathbb{S}^{e}\right)^{T}=-\mathbb{S}^{e} .
$$

Then, one can obtain

$$
\Theta^{r, n+1}-\Theta^{r, n}=0
$$

which completes the proof.

Theorem 2. (CFL condition) Using the POD-DGTD scheme (15)-(16) when $\Gamma_{a}=\varnothing$, the global discrete electromagnetic energy (18) is a positive definite quadratic form of the unknowns $\underline{\mathbf{E}}_{h}^{r}$ and $\underline{\mathbf{H}}_{h}^{r}$ if

$$
\Delta t<\frac{2}{d_{N}^{r}}, \text { with } d_{N}^{r}=\left\|\left(\Psi_{\mathbf{H}}^{T} \mathbb{M}^{\mu_{r}} \Psi_{\mathbf{H}}\right)^{-\frac{1}{2}} \Psi_{\mathbf{H}}^{T}\left(\mathbb{K}-\mathbb{S}^{i}-\mathbb{S}^{e}\right) \Psi_{\mathbf{E}}\left(\Psi_{\mathbf{E}}^{T} \mathbb{M}^{\varepsilon_{r}} \Psi_{\mathbf{E}}\right)^{-\frac{1}{2}}\right\| \text {. }
$$

Proof. Using the scheme (16) to develop $\alpha_{\mathbf{H}}^{\left(n+\frac{1}{2}\right)}$ in function of $\alpha_{\mathbf{H}}^{\left(n-\frac{1}{2}\right)}$ and $\alpha_{\mathbf{E}}^{(n)}$, one can get

$$
\begin{array}{r}
\Theta^{r, n}=\frac{1}{2}\left(\alpha_{\mathbf{E}}^{(n)}\right)^{T}\left(\Psi_{\mathbf{E}}^{T} \mathbb{M}^{\varepsilon_{r}} \Psi_{\mathbf{E}}\right) \alpha_{\mathbf{E}}^{(n)}+\frac{1}{2}\left(\alpha_{\mathbf{H}}^{\left(n-\frac{1}{2}\right)}\right)^{T}\left(\Psi_{\mathbf{H}}^{T} \mathbb{M}^{\mu_{r}} \Psi_{\mathbf{H}}\right) \alpha_{\mathbf{H}}^{\left(n+\frac{1}{2}\right)} \\
=\frac{1}{2}\left(\alpha_{\mathbf{E}}^{(n)}\right)^{T}\left(\Psi_{\mathbf{E}}^{T} \mathbb{M}^{\varepsilon_{r}} \Psi_{\mathbf{E}}\right) \alpha_{\mathbf{E}}^{(n)}+\frac{1}{2}\left(\alpha_{\mathbf{H}}^{\left(n-\frac{1}{2}\right)}\right)^{T}\left(\Psi_{\mathbf{H}}^{T} \mathbb{M}^{\mu_{r}} \Psi_{\mathbf{H}}\right) \alpha_{\mathbf{H}}^{\left(n-\frac{1}{2}\right)} \\
-\frac{\Delta t}{2}\left(\alpha_{\mathbf{H}}^{\left(n-\frac{1}{2}\right)}\right)^{T} \Psi_{\mathbf{H}}^{T}\left(\mathbb{K}-\mathbb{S}^{i}-\mathbb{S}^{e}\right) \Psi_{\mathbf{E}} \alpha_{\mathbf{E}}^{(n)} .
\end{array}
$$

We have the following equalities and inequalities

$$
\begin{aligned}
& \frac{1}{2}\left(\alpha_{\mathbf{E}}^{(n)}\right)^{T}\left(\Psi_{\mathbf{E}}^{T} \mathbb{M}^{\varepsilon_{r}} \Psi_{\mathbf{E}}\right) \alpha_{\mathbf{E}}^{(n)}=\frac{1}{2}\left\|\left(\Psi_{\mathbf{E}}^{T} \mathbb{M}^{\varepsilon_{r}} \Psi_{\mathbf{E}}\right)^{\frac{1}{2}} \alpha_{\mathbf{E}}^{(n)}\right\|^{2}, \\
& \frac{1}{2}\left(\alpha_{\mathbf{H}}^{\left(n-\frac{1}{2}\right)}\right)^{T}\left(\Psi_{\mathbf{H}}^{T} \mathbb{M}^{\mu_{r}} \Psi_{\mathbf{H}}\right) \alpha_{\mathbf{H}}^{\left(n-\frac{1}{2}\right)}=\frac{1}{2}\left\|\left(\Psi_{\mathbf{H}}^{T} \mathbb{M}^{\mu_{r}} \Psi_{\mathbf{H}}\right)^{\frac{1}{2}} \alpha_{\mathbf{H}}^{\left(n-\frac{1}{2}\right)}\right\|^{2}, \\
& \frac{\Delta t}{2}\left(\alpha_{\mathbf{H}}^{\left(n-\frac{1}{2}\right)}\right)^{T} \Psi_{\mathbf{H}}^{T}\left(\mathbb{K}-\mathbb{S}^{i}-\mathbb{S}^{e}\right) \Psi_{\mathbf{E}} \alpha_{\mathbf{E}}^{(n)} \leq \mid \frac{\Delta t}{2}\left(\alpha_{\mathbf{H}}^{\left(n-\frac{1}{2}\right)}\right)^{T}\left(\Psi_{\mathbf{H}}^{T} \mathbb{M}^{\mu_{r}} \Psi_{\mathbf{H}}\right)^{\frac{1}{2}}\left(\Psi_{\mathbf{H}}^{T} \mathbb{M}^{\mu_{r}} \Psi_{\mathbf{H}}\right)^{-\frac{1}{2}} \\
& \Psi_{\mathbf{H}}^{T} \quad\left(\mathbb{K}-\mathbb{S}^{i}-\mathbb{S}^{e}\right) \Psi_{\mathbf{E}}\left(\Psi_{\mathbf{E}}^{T} \mathbb{M}^{\varepsilon_{r}} \Psi_{\mathbf{E}}\right)^{-\frac{1}{2}}\left(\Psi_{\mathbf{E}}^{T} \mathbb{M}^{\varepsilon_{r}} \Psi_{\mathbf{E}}\right)^{\frac{1}{2}} \alpha_{\mathbf{E}}^{(n)} \mid \\
& \leq \frac{\Delta t d_{N}^{r}}{2}\left\|\left(\Psi_{\mathbf{E}}^{T} \mathbb{M}^{\varepsilon_{r}} \Psi_{\mathbf{E}}\right)^{\frac{1}{2}} \alpha_{\mathbf{E}}^{(n)}\right\|\left\|\left(\Psi_{\mathbf{H}}^{T} \mathbb{M}^{\mu_{r}} \Psi_{\mathbf{H}}\right)^{\frac{1}{2}} \alpha_{\mathbf{H}}^{\left(n-\frac{1}{2}\right)}\right\|
\end{aligned}
$$

Noticing that

$$
\left\|\left(\Psi_{\mathbf{E}}^{T} \mathbb{M}^{\varepsilon_{r}} \Psi_{\mathbf{E}}\right)^{\frac{1}{2}} \alpha_{\mathbf{E}}^{(n)}\right\|\left\|\left(\Psi_{\mathbf{H}}^{T} \mathbb{M}^{\mu_{r}} \Psi_{\mathbf{H}}\right)^{\frac{1}{2}} \alpha_{\mathbf{H}}^{\left(n-\frac{1}{2}\right)}\right\| \leq \frac{1}{2}\left(\left\|\left(\Psi_{\mathbf{E}}^{T} \mathbb{M}^{\varepsilon_{r}} \Psi_{\mathbf{E}}\right)^{\frac{1}{2}} \alpha_{\mathbf{E}}^{(n)}\right\|^{2}+\left\|\left(\Psi_{\mathbf{H}}^{T} \mathbb{M}^{\mu_{r}} \Psi_{\mathbf{H}}\right)^{\frac{1}{2}} \alpha_{\mathbf{H}}^{\left(n-\frac{1}{2}\right)}\right\|^{2}\right),
$$

then one can get

$$
\Theta^{r, n} \geq \frac{1}{2}\left(1-\frac{\Delta t d_{N}^{r}}{2}\right)\left\|\left(\Psi_{\mathbf{E}}^{T} \mathbb{M}^{\varepsilon_{r}} \Psi_{\mathbf{E}}\right)^{\frac{1}{2}} \alpha_{\mathbf{E}}^{(n)}\right\|^{2}+\frac{1}{2}\left(1-\frac{\Delta t d_{N}^{r}}{2}\right)\left\|\left(\Psi_{\mathbf{H}}^{T} \mathbb{M}^{\mu_{r}} \Psi_{\mathbf{H}}\right)^{\frac{1}{2}} \alpha_{\mathbf{H}}^{\left(n-\frac{1}{2}\right)}\right\|^{2} .
$$

This concludes the proof. Expression (22) states a sufficient condition for the stability of the fully discrete reduced-order model (16). 


\subsection{Error estimates}

The error estimate for the ROM (12) and (16) will be used as an error control mechanism in the adaptive snapshot selection algorithm. The error $\mathbf{e}_{\mathbf{u}}(t)=\underline{\mathbf{u}}_{h}-\underline{\mathbf{u}}_{h}^{r}(\mathbf{u}=\mathbf{E}, \mathbf{H})$ over the time interval $\left[0, T_{f}\right]$ is decomposed as

$$
\mathbf{e}_{\mathbf{u}}(t)=\mathbf{e}_{\mathbf{u}, P}(t)+\mathbf{e}_{\mathbf{u}, V}(t),
$$

with

$$
\left\{\begin{array}{l}
\mathbf{e}_{\mathbf{u}, P}(t)=\left[\mathbb{I}-\Psi_{\mathbf{u}}\left(\Psi_{\mathbf{u}}^{T} \mathbb{M} \Psi_{\mathbf{u}}\right)^{-1} \Psi_{\mathbf{u}}^{T} \mathbb{M}\right] \underline{\mathbf{u}}_{h} \\
\mathbf{e}_{\mathbf{u}, V}(t)=\Psi_{\mathbf{u}}\left[\left(\Psi_{\mathbf{u}}^{T} \mathbb{M} \Psi_{\mathbf{u}}\right)^{-1} \Psi_{\mathbf{u}}^{T} \mathbb{M} \underline{\mathbf{u}}_{h}-\alpha_{\mathbf{u}}(t)\right]
\end{array}\right.
$$

We decompose $\mathcal{R}^{N}$ as

$$
\mathcal{R}^{N}=\operatorname{span} \Psi_{\mathbf{u}} \oplus^{\perp \mathbb{M}} \operatorname{span} \Phi_{\mathbf{u}}, \quad \mathbf{u}=\mathbf{E}, \mathbf{H},
$$

where span $\mathbb{A}$ denotes the subspace spanned by the columns of matrix $\mathbb{A}$, and ${ }^{\perp \mathbb{M}}$ means that $\operatorname{span} \Phi_{\mathbf{u}}$ and $\operatorname{span} \Psi_{\mathbf{u}}$ are orthogonal with respect to $\mathbb{M}$, that is $\Phi_{\mathbf{u}}^{T} \mathbb{M} \Psi_{\mathbf{u}}=0$. We can easily find that the error components $\mathbf{e}_{\mathbf{u}, P}(t)$ and $\mathbf{e}_{\mathbf{u}, V}(t)$ belong to $\operatorname{span} \Phi_{\mathbf{u}}$ and $\operatorname{span} \Psi_{\mathbf{u}}$, respectively.

We first introduce two norms and two operators in order to derive an estimation for the error. For a matrix $\mathbb{K}$, the $\mathbb{K}$-norm of a vector $\mathbf{x}$ and a matrix $\mathbb{Y}$ can be defined as

$$
\|\mathbf{x}\|_{\mathbb{K}}=\sqrt{\mathbf{x}^{T} \mathbb{K} \mathbf{x}}, \quad\|\mathbb{Y}\|_{\mathbb{K}}=\max _{\mathbf{x} \neq 0} \frac{\|\mathbb{Y} \mathbf{x}\|_{\mathbb{K}}}{\|\mathbf{x}\|_{\mathbb{K}}}, \quad \forall \mathbb{Y} \in \mathcal{R}^{N \times N}, \forall \mathbf{x} \in \mathcal{R}^{N} .
$$

When the matrix $\mathbb{K}$ is a symmetric positive definite matrix, the matrix norm $\|\mathbb{Y}\|_{\mathbb{K}}$ is $\sqrt{\lambda_{\max }\left(\mathbb{Y}^{T} \mathbb{K} \mathbb{Y}\right)}$, where $\lambda_{\max }\left(\mathbb{Y}^{T} \mathbb{K} \mathbb{Y}\right)$ denotes the maximum eigenvalue of the matrix $\mathbb{Y}^{T} \mathbb{K} \mathbb{Y}$. Besides, as in [13], the operators $F\left(T_{f} ; \mathbb{Y}\right): L^{2}\left(\left[0, T_{f}\right], \mathcal{R}^{N}\right) \rightarrow L^{2}\left(\left[0, T_{f}\right], \mathcal{R}^{N}\right)$ and $G\left(T_{f} ; \mathbb{Y}\right): \mathcal{R}^{N} \rightarrow L^{2}\left(\left[0, T_{f}\right], \mathcal{R}^{N}\right)$ are linear operators if the integral equation

$$
\mathbf{x}(t)=\int_{0}^{t} \mathrm{e}^{\mathbb{Y}(t-\tau)} \mathbf{u}(\tau) d \tau+\mathrm{e}^{\mathbb{Y} t} \mathbf{x}_{0}, \quad \mathbb{Y} \in \mathcal{R}^{N \times N}, \mathbf{x}, \mathbf{x}_{0}, \mathbf{u} \in \mathcal{R}^{N},
$$

is written as the form

$$
\mathbf{x}=F\left(T_{f} ; \mathbb{Y}\right) \mathbf{u}+G\left(T_{f} ; \mathbb{Y}\right) \mathbf{x}_{0},
$$

in the interval $\left[0, T_{f}\right]$. For complete descriptions of the definition of the operators $F\left(T_{f} ; \mathbb{Y}\right)$ and $G\left(T_{f} ; \mathbb{Y}\right)$ see $[3,13]$. In this paper, we shall estimate $\|\mathbf{x}\|_{\mathbb{K}}$ based on the matrix theory

$$
\|\mathbf{x}\|_{\mathbb{K}} \leq\left\|F\left(T_{f} ; \mathbb{Y}\right)\right\|_{\mathbb{K}}\|\mathbf{u}\|_{\mathbb{K}}+\left\|G\left(T_{f} ; \mathbb{Y}\right)\right\|_{\mathbb{K}}\left\|\mathbf{x}_{0}\right\|_{\mathbb{K}} .
$$

Lemma 1. [3] Given a sequence $\left\{\phi_{n}\right\}_{n=0}^{N_{t}}$ such that

$$
0 \leq \phi_{n} \leq \beta \phi_{n-1}+\alpha \Delta t \psi_{n}+\alpha \Delta t \psi_{n-1}, \quad \text { for } n \geq 1,
$$

where $\beta>0, \alpha \geq 0$, and $\psi_{n} \geq 0\left(n=0,1, \cdots, N_{t}\right)$. For $n=0,1, \cdots, N_{t}$, $\phi_{n}$ satisfies

$$
\phi_{n}^{2} \leq 2 \beta^{2 n} \phi_{0}+2\left[\alpha \Delta t\left(1+\frac{1}{\beta}\right)\right]^{2}\left(\sum_{i=0}^{N_{t}} \beta^{2 i}\right)\left(\sum_{i=0}^{N_{t}} \psi_{i}^{2}\right),
$$

and

$$
\Delta t \sum_{j=0}^{N_{t}} \phi_{j}^{2} \leq 2 \phi_{0} \sum_{j=0}^{N_{t}} \beta^{2 j}+2 T_{f}\left[\alpha\left(1+\frac{1}{\beta}\right)\right]^{2}\left(\Delta t \sum_{i=0}^{N_{t}} \beta^{2 i}\right)\left(\Delta t \sum_{i=0}^{N_{t}} \psi_{i}^{2}\right) .
$$

Proof. Proceeding by induction, one can obtain

$$
\phi_{n} \leq \beta^{n} \phi_{0}+\alpha \Delta t \psi_{n}+\alpha \Delta t(1+\beta) \sum_{i=0}^{n-1} \beta^{n-1-i} \psi_{i}, \text { for } n \geq 1 .
$$

Equation (33) can be rewritten as follows (because $1+\frac{1}{\beta}>1$ )

$$
\phi_{n} \leq \beta^{n} \phi_{0}+\alpha \Delta t\left(1+\frac{1}{\beta}\right) \sum_{i=0}^{n} \beta^{n-i} \psi_{i} .
$$

The lemma is proved by squaring the inequality (34) and then using the Cauchy-Schwarz inequality. 
Theorem 3. Let $\underline{\mathbf{U}}_{h}(t)=\left(\underline{\mathbf{E}}_{h}(t), \underline{\mathbf{H}}_{h}(t)\right)$ ( $\left.t \in\left[0, T_{f}\right]\right)$ be the semi-discrete DG solution of (5), and $\underline{\mathbf{U}}_{h}^{r}(t)=\left(\underline{\mathbf{E}}_{h}^{r}(t), \underline{\mathbf{H}}_{h}^{r}(t)\right)\left(t \in\left[0, T_{f}\right]\right)$ be the reduced-order solution of (10), and (12). Then, the error $\mathbf{e}_{R O M}(t)=\underline{\mathbf{U}}_{h}(t)-\underline{\mathbf{U}}_{h}^{r}(t)\left(t \in\left[0, T_{f}\right]\right)$ for the POD-based ROM in the semi-discrete setting satisfies

$$
\begin{array}{r}
\int_{0}^{T_{f}}\left\|\mathbf{e}_{R O M}(t)\right\|_{\widehat{\mathbb{M}}}^{2} d t \leq\left(\left\|F\left(T_{f} ;\left(\widehat{\Psi}^{T} \widehat{\mathbb{M}} \widehat{\Psi}\right)^{-1} \widehat{\Psi}^{T} \mathbb{R} \widehat{\Psi}\right)\right\|_{\widehat{\Psi}^{T} \widehat{\mathbb{M}} \widehat{\Psi}}^{2}\left\|\widehat{\Psi}^{T} \mathbb{R} \widehat{\Phi}\left(\widehat{\Phi}^{T} \widehat{\mathbb{M}} \widehat{\Phi}\right)^{-1} \widehat{\Phi}^{T} \widehat{\mathbb{M}}^{\frac{1}{2}}\right\|_{(\widehat{\Psi}}^{2} \widehat{\mathbb{M} \widehat{\Psi})^{-1}}+1\right) \\
\times\left(\int_{0}^{T_{f}}\left\|\mathbf{e}_{\mathbf{E}, P}(t)\right\|_{\mathbb{M}}^{2} d t+\int_{0}^{T_{f}}\left\|\mathbf{e}_{\mathbf{H}, P}(t)\right\|_{\mathbb{M}}^{2} d t\right)
\end{array}
$$

with

$\widehat{\Psi}=\left[\begin{array}{cc}\Psi_{\mathbf{E}} & 0 \\ 0 & \Psi_{\mathbf{H}}\end{array}\right], \widehat{\Phi}=\left[\begin{array}{cc}\Phi_{\mathbf{E}} & 0 \\ 0 & \Phi_{\mathbf{H}}\end{array}\right], \widehat{\mathbb{M}}=\left[\begin{array}{cc}\mathbb{M} & 0 \\ 0 & \mathbb{M}\end{array}\right]$, and $\mathbb{R}=\left[\begin{array}{cc}0 & \varepsilon_{r}^{-1}\left(\mathbb{K}-\mathbb{S}^{i}-\mathbb{S}^{h}\right) \\ \mu_{r}^{-1}\left(-\mathbb{K}+\mathbb{S}^{i}+\mathbb{S}^{e}\right) & 0\end{array}\right]$ where $\widehat{\mathbb{M}} \frac{1}{2}$ is the square root of the real symmetric positive definite matrix $\widehat{\mathbb{M}}$.

The proof of THEOREM 3 is provided in appendix Appendix A. We now consider the error bound for the fully discrete system. Similarly, the error $\mathbf{e}_{\mathbf{E}}^{(n)}=\underline{\mathbf{E}}_{h}^{(n)}-\underline{\mathbf{E}}_{h}^{r,(n)}$ is decomposed as

$$
\mathbf{e}_{\mathbf{E}}^{(n)}=\mathbf{e}_{\mathbf{E}, P}^{(n)}+\mathbf{e}_{\mathbf{E}, V}^{(n)}, \quad n=0,1, \cdots, N_{t},
$$

with

$$
\left\{\begin{array}{l}
\mathbf{e}_{\mathbf{E}, P}^{(n)}=\left[\mathbb{I}-\Psi_{\mathbf{E}}\left(\Psi_{\mathbf{E}}^{T} \mathbb{M} \Psi_{\mathbf{E}}\right)^{-1} \Psi_{\mathbf{E}}^{T} \mathbb{M}\right] \underline{\mathbf{E}}_{h}^{(n)} \in \operatorname{span} \Phi_{\mathbf{E}} \\
\mathbf{e}_{\mathbf{E}, V}^{(n)}=\Psi_{\mathbf{E}}\left[\left(\Psi_{\mathbf{E}}^{T} \mathbb{M} \Psi_{\mathbf{E}}\right)^{-1} \Psi_{\mathbf{E}}^{T} \mathbb{M}_{h}^{(n)}-\alpha_{\mathbf{E}}^{(n)}\right] \in \operatorname{span} \Psi_{\mathbf{E}}
\end{array}\right.
$$

The error $\mathbf{e}_{\mathbf{H}}^{\left(n+\frac{1}{2}\right)}$ is also decomposed as

$$
\mathbf{e}_{\mathbf{H}}^{\left(n+\frac{1}{2}\right)}=\mathbf{e}_{\mathbf{H}, P}^{\left(n+\frac{1}{2}\right)}+\mathbf{e}_{\mathbf{H}, V}^{\left(n+\frac{1}{2}\right)}, \quad n=0,1, \cdots, N_{t},
$$

with

$$
\left\{\begin{array}{l}
\mathbf{e}_{\mathbf{H}, P}^{\left(n+\frac{1}{2}\right)}=\left[\mathbb{I}-\Psi_{\mathbf{H}}\left(\Psi_{\mathbf{H}}^{T} \mathbb{M} \Psi_{\mathbf{H}}\right)^{-1} \Psi_{\mathbf{H}}^{T} \mathbb{M}\right] \underline{\mathbf{H}}_{h}^{\left(n+\frac{1}{2}\right)} \in \operatorname{span} \Phi_{\mathbf{H}}, \\
\mathbf{e}_{\mathbf{H}, V}^{\left(n+\frac{1}{2}\right)}=\Psi_{\mathbf{H}}\left[\left(\Psi_{\mathbf{H}}^{T} \mathbb{M} \Psi_{\mathbf{H}}\right)^{-1} \Psi_{\mathbf{H}}^{T} \mathbb{M} \underline{\mathbf{H}}_{h}^{\left(n+\frac{1}{2}\right)}-\alpha_{\mathbf{H}}^{\left(n+\frac{1}{2}\right)}\right] \in \operatorname{span} \Psi_{\mathbf{H}}
\end{array}\right.
$$

Theorem 4. Let $\underline{\mathbf{U}}_{h}^{(n)}=\left(\underline{\mathbf{E}}_{h}^{(n)}, \underline{\mathbf{H}}_{h}^{\left(n+\frac{1}{2}\right)}\right)\left(n=0,1, \cdots, N_{t}\right)$ be the DGTD solution of (7), and $\underline{\mathbf{U}}_{h}^{r,(n)}$ $=\left(\underline{\mathbf{E}}_{h}^{r,(n)}, \underline{\mathbf{H}}_{h}^{r,\left(n+\frac{1}{2}\right)}\right)\left(n=0,1, \cdots, N_{t}\right)$ be the reduced-order solution of (16). Then, the error $\mathbf{e}_{R O M}^{(j)}=$ $\underline{\mathbf{U}}_{h}^{(n)}-\underline{\mathbf{U}}_{h}^{r,(n)}\left(n=0,1, \cdots, N_{t}\right)$ for the ROM in the discrete system satisfies

$$
\Delta t \sum_{j=0}^{N_{t}}\left\|\mathbf{e}_{R O M}^{(j)}\right\|_{\widehat{\mathbb{M}}}^{2} \leq\left(1+2 T_{f}\left[\alpha\left(1+\frac{1}{\beta}\right)\right]^{2}\left(\Delta t \sum_{j=0}^{N_{t}} e^{2 \gamma j \Delta t}\right)\right) \times\left(\Delta t \sum_{i=0}^{N_{t}}\left(\left\|\mathbf{e}_{\mathbf{E}, P}^{(i)}\right\|_{\mathbb{M}}+\left\|\mathbf{e}_{\mathbf{H}, P}^{\left(i+\frac{1}{2}\right)}\right\|_{\mathbb{M}}\right)^{2}\right)
$$

with

$$
\left\{\begin{aligned}
\alpha & =\max \left\{\alpha_{1}, \alpha_{2}\right\} \\
\beta & =\| \mathbb{I}+\Delta t \mathbb{M} \frac{1}{2}\left\{\widehat{\Psi}\left(\widehat{\Psi}^{T} \widehat{\mathbb{M}} \widehat{\Psi}\right)^{-1} \widehat{\Psi}^{T} \mathbb{R}\right. \\
& \left.+\Delta t\left[\widehat{\Psi}\left(\widehat{\Psi}^{T} \widehat{\mathbb{M}} \widehat{\Psi}\right)^{-1} \widehat{\Psi}^{T} \mathbb{L}\right]\left[\widehat{\Psi}\left(\widehat{\Psi}^{T} \widehat{\mathbb{M}} \widehat{\Psi}\right)^{-1} \widehat{\Psi}^{T} \mathbb{U}\right]\right\} \mathbb{M}^{-\frac{1}{2}} \|_{2} \\
\gamma & =\| \mathbb{M} \frac{1}{2}\left\{\widehat{\Psi}\left(\widehat{\Psi}^{T} \widehat{\mathbb{M}} \widehat{\Psi}\right)^{-1} \widehat{\Psi}^{T} \mathbb{R}\right. \\
& \left.+\Delta t\left[\widehat{\Psi}\left(\widehat{\Psi}^{T} \widehat{\mathbb{M}} \widehat{\Psi}\right)^{-1} \widehat{\Psi}^{T} \mathbb{L}\right]\left[\widehat{\Psi}\left(\widehat{\Psi}^{T} \widehat{\mathbb{M}} \widehat{\Psi}\right)^{-1} \widehat{\Psi}^{T} \mathbb{U}\right]\right\} \mathbb{M}^{-\frac{1}{2}} \|_{2},
\end{aligned}\right.
$$

where $\alpha_{1}, \alpha_{2}, \mathbb{L}$, and $\mathbb{U}$ are respectively defined as

$$
\left\{\begin{array}{l}
\alpha_{1}=\left\|\widehat{\mathbb{M}}^{\frac{1}{2}}\left[\mathbb{I}+\Delta t \widehat{\Psi}\left(\widehat{\Psi}^{T} \widehat{\mathbb{M}} \widehat{\Psi}\right)^{-1} \widehat{\Psi}^{T} \mathbb{L}\right] \widehat{\Psi}\left(\widehat{\Psi}^{T} \widehat{\mathbb{M}} \widehat{\Psi}\right)^{-1} \widehat{\Psi}^{T} \mathbb{U} \widehat{\Phi}\left(\widehat{\Phi}^{T} \widehat{\mathbb{M}} \widehat{\Phi}\right)^{-1} \widehat{\Phi}^{T} \widehat{\mathbb{M}}^{\frac{1}{2}}\right\|_{2}, \\
\alpha_{2}=\left\|\widehat{\mathbb{M}}^{\frac{1}{2}}\left[\mathbb{I}+\Delta t \widehat{\Psi}\left(\widehat{\Psi}^{T} \widehat{\mathbb{M}} \widehat{\Psi}\right)^{-1} \widehat{\Psi}^{T} \mathbb{L}\right] \widehat{\Psi}\left(\widehat{\Psi}^{T} \widehat{\mathbb{M}} \widehat{\Psi}\right)^{-1} \widehat{\Psi}^{T} \mathbb{L} \widehat{\Phi}\left(\widehat{\Phi}^{T} \widehat{\mathbb{M}} \widehat{\Phi}\right)^{-1} \widehat{\Phi}^{T} \widehat{\mathbb{M}}^{\frac{1}{2}}\right\|_{2},
\end{array}\right.
$$$$
\mathbb{L}=\left[\begin{array}{cc}
0 & 0 \\
\mu_{r}^{-1}\left(-\mathbb{K}+\mathbb{S}^{i}+\mathbb{S}^{e}\right) & 0
\end{array}\right], \mathbb{U}=\left[\begin{array}{cc}
0 & \varepsilon_{r}^{-1}\left(\mathbb{K}-\mathbb{S}^{i}-\mathbb{S}^{h}\right) \\
0 & 0
\end{array}\right]
$$ 
The proof of THEOREM 4 is provided in appendix Appendix B.

Reamrk 3. The error estimate of the POD-based ROM in the semi-discrete level (THEOREM 3) for the system of time-domain Maxwell equations discretized by the DG method is not fully novel but used to come to a good snapshot selection, and is very similar to the ones initially derived in [3], in which the error bound for the first-order dynamical system is derived at the semi-discrete level. However, the discrete system was obtained after time integration with the average constant acceleration Newmark scheme is analyzed in [3], while, the error bound for POD-DGTD scheme is derived in the discrete level when the $\mathrm{LF}_{2}$ time scheme is applied in THEOREM 4.

Reamrk 4. From TheOREMS 3 and 4 , the total errors $\mathbf{e}_{R O M}(t)\left(t \in\left[0, T_{f}\right]\right)$ and $\mathbf{e}_{R O M}^{(j)}\left(j=0,1, \cdots, N_{t}\right)$ are only controlled by the errors projected onto the subspace spanned by $\Phi_{\mathbf{u}}(\mathbf{u}=\mathbf{E}, \mathbf{H})$, i.e., one can estimate the errors for a given set of snapshots without knowing the solutions of the ROM. These error estimates will be used as the error control mechanism in the adaptive selection algorithm for choosing snapshots, and to guarantee the low-rank property of the snapshot matrix $\mathbb{A}_{\mathbf{u}}(\mathbf{u}=\mathbf{E}, \mathbf{H})$. This technique is described in the subsequent section.

Reamrk 5. In the numerical simulations, one does not need to calculate the coefficients in THEOREM 3 and 4 , e.g. $\alpha, \beta$ and $\gamma$. Instead, one needs to know that the errors are only controlled by the errors projected onto the subspace $\operatorname{span} \Phi_{\mathbf{u}}(\mathbf{u}=\mathbf{E}, \mathbf{H})$.

\section{An adaptive algorithm}

In this section, an ISVD algorithm for computing the singular vectors on-the-fly [45], and an adaptive selection algorithm for choosing snapshots are represented.

\subsection{Incremental $S V D$}

The ISVD algorithm is an on-the-fly algorithm of the SVD [45]. The underlying idea is to update the POD basis as soon as a new snapshot is added [46]. In this paper, a modified version of Brand's ISVD [42] proposed by Oxberry, et al. is adopted because of its simplicity in implementation and description [1]. A rank- $k$ SVD of a matrix $\mathbb{A}_{\mathbf{u}}$ is defined by

$$
\mathbb{A}_{\mathbf{u}}=\mathbb{U}_{\mathbf{u}, k} \Sigma_{k \times k}^{\mathbf{u}} \mathbb{V}_{\mathbf{u}, k}^{T}+\mathbb{R}_{\mathbf{u}}, \quad k \leq r_{\mathbf{u}} \leq \min \{N, \ell\},
$$

where $\mathbb{U}_{\mathbf{u}, k} \Sigma_{k \times k}^{u} \mathbb{V}_{\mathbf{u}, k}^{T}$ is the rank- $k$ SVD similar to (9), $\mathbb{R}_{\mathbf{u}} \in \mathcal{R}^{N \times \ell}$ is the error because of rank- $k$ truncation, which equals to 0 when $k=r_{\mathbf{u}}$. The rank- $k$ SVD matrix is updated when a new snapshot $\mathbf{u}_{h}^{n_{\text {new }}}$ is available. The POD snapshot matrix is a low-rank matrix, so we choose $k=r_{\mathbf{u}}$ in this work. This ISVD algorithm is derived from the identity

$$
\begin{aligned}
{\left[\mathbb{A}_{\mathbf{u}}, \mathbf{u}_{h}^{n_{\text {new }}}\right] } & =\left[\mathbb{U}_{\mathbf{u}, k} \Sigma_{k \times k}^{\mathbf{u}} \mathbb{V}_{\mathbf{u}, k}^{T}, \mathbf{u}_{h}^{n_{\text {new }}}\right] \\
& =\left[\mathbb{U}_{\mathbf{u}, k},\left(\mathbb{I}-\mathbb{U}_{\mathbf{u}, k} \mathbb{U}_{\mathbf{u}, k}^{T}\right) \mathbf{u}_{h}^{n_{\text {new }}} / p\right]\left[\begin{array}{cc}
\Sigma_{k \times k}^{\mathbf{u}} & \mathbb{U}_{\mathbf{u}, k}^{T} \mathbf{u}_{h}^{n_{\text {new }}} \\
0_{1 \times k} & p
\end{array}\right]\left[\begin{array}{cc}
\mathbb{V}_{\mathbf{u}, k} & 0_{k \times 1} \\
0_{1 \times k} & 1
\end{array}\right]^{T} \\
& =\left[\mathbb{U}_{\mathbf{u}, k}, \mathbf{q}\right]\left[\begin{array}{cc}
\Sigma_{k \times k}^{\mathbf{u}} & \mathbf{1} \\
0_{1 \times k} & p
\end{array}\right]\left[\begin{array}{cc}
\mathbb{V}_{\mathbf{u}, k} & 0_{k \times 1} \\
0_{1 \times k} & 1
\end{array}\right]^{T},
\end{aligned}
$$

where $p=\left\|\mathbf{u}_{h}^{n_{\text {new }}}-\mathbb{U}_{\mathbf{u}, k} \mathbb{U}_{\mathbf{u}, k}^{T} \mathbf{u}_{h}^{n_{\text {new }}}\right\|$ is the length of the orthogonal projection of $\mathbf{u}_{h}^{n_{\text {new }}}$ onto the subspace with an orthogonal basis $\mathbb{U}_{\mathbf{u}, k}, \mathbf{q}=\left(\mathbb{I}-\mathbb{U}_{\mathbf{u}, k} \mathbb{U}_{\mathbf{u}, k}^{T}\right) \mathbf{u}_{h}^{n_{\text {new }}} / p$ is the normalized orthogonal projection of $\mathbf{u}_{h}^{n_{\text {new }}}$ onto the subspace with an orthogonal basis $\mathbb{U}_{\mathbf{u}, k}$, and $\mathbf{l}=\mathbb{U}_{\mathbf{u}, k}^{T} \mathbf{u}_{h}^{n_{\text {new }}}$ is the projection $\mathbf{u}_{h}^{n_{\text {new }}}$ onto the orthogonal basis $\mathbb{U}_{\mathbf{u}, k}$. The matrix in the middle, which will be denoted by $\mathbb{Q}$, must be diagonalized to update the SVD

Then the updated SVD is

$$
\mathbb{Q}=\left[\begin{array}{cc}
\Sigma_{k \times k}^{\mathbf{u}} & \mathbf{1} \\
0_{1 \times k} & p
\end{array}\right]=\widehat{\mathbb{U}}_{\mathbf{u}, k} \widehat{\Sigma}_{k \times k}^{\mathbf{u}} \widehat{\mathbb{V}}_{\mathbf{u}, k}^{T}
$$

$$
\begin{aligned}
{\left[\mathbb{A}_{\mathbf{u}}, \mathbf{u}_{h}^{n_{\text {new }}}\right] } & =\left[\mathbb{U}_{\mathbf{u}, k}, \mathbf{q}\right] \widehat{\mathbb{U}}_{\mathbf{u}, k} \widehat{\Sigma}_{k \times k}^{\mathbf{u}} \widehat{\mathbb{V}}_{\mathbf{u}, k}^{T}\left[\begin{array}{cc}
\mathbb{V}_{\mathbf{u}, k} & 0_{k \times 1} \\
0_{1 \times k} & 1
\end{array}\right]^{T} \\
& =\mathbb{U}_{\mathbf{u}}^{\text {new }} \Sigma^{\mathbf{u}, \text { new }} \mathbb{V}_{\mathbf{u}}^{\text {new } T}
\end{aligned}
$$


where $\mathbb{U}_{\mathbf{u}}^{\text {new }}=\left[\mathbb{U}_{\mathbf{u}, k}, \mathbf{q}\right] \widehat{\mathbb{U}}_{\mathbf{u}, k}, \Sigma^{\mathbf{u}, \text { new }}=\widehat{\Sigma}_{k \times k}^{\mathbf{u}}$, and $\mathbb{V}_{\mathbf{u}}^{\text {new }}=\left[\begin{array}{cc}\mathbb{V}_{\mathbf{u}, k} & 0_{k \times 1} \\ 0_{1 \times k} & 1\end{array}\right] \widehat{\mathbb{V}}_{\mathbf{u}, k}$. The SVD matrices are initialized by the following formulations

$$
\mathbb{U}_{\mathbf{u}}^{\text {init }}=\mathbf{u}_{h}^{n_{1}} /\left\|\mathbf{u}_{h}^{n_{1}}\right\|, \Sigma^{\mathbf{u}, \text { init }}=\left\|\mathbf{u}_{h}^{n_{1}}\right\|, \quad \mathbb{V}_{\mathbf{u}}^{\text {init }}=1 .
$$

In particular, an SVD truncation tolerance $\varepsilon_{\text {SVD }}$ is used to determine if the new snapshot $\mathbf{u}_{h}^{n_{\text {new }}}$ is numerically linearly independent on the columns of $\mathbb{A}_{\mathbf{u}}$. If $p<\varepsilon_{\mathrm{SVD}}$, (42) can be replaced with the truncated forms

$$
\mathbb{U}_{\mathbf{u}}^{\text {new }}=\mathbb{U}_{\mathbf{u}, k} \widehat{\mathbb{U}}_{\mathbf{u}, k_{1: r, 1: r}}, \Sigma^{\mathbf{u}, \text { new }}=\widehat{\Sigma}_{1: r, 1: r}^{\mathbf{u}}, \mathbb{V}_{\mathbf{u}}^{\text {new }}=\mathbb{V}_{\mathbf{u}, k} \widehat{\mathbb{V}}_{\mathbf{u}, k_{:, 1: r}} .
$$

We refer the reader to $[42,45]$ for more details. In this paper, we use the ISVD algorithm to compute the singular vectors and singular values of the augmented snapshot matrix $\mathbb{A}_{\mathbf{u}}(\mathbf{u}=\mathbf{E}, \mathbf{H})$ on-the-fly, which is described in Algorithm 1.

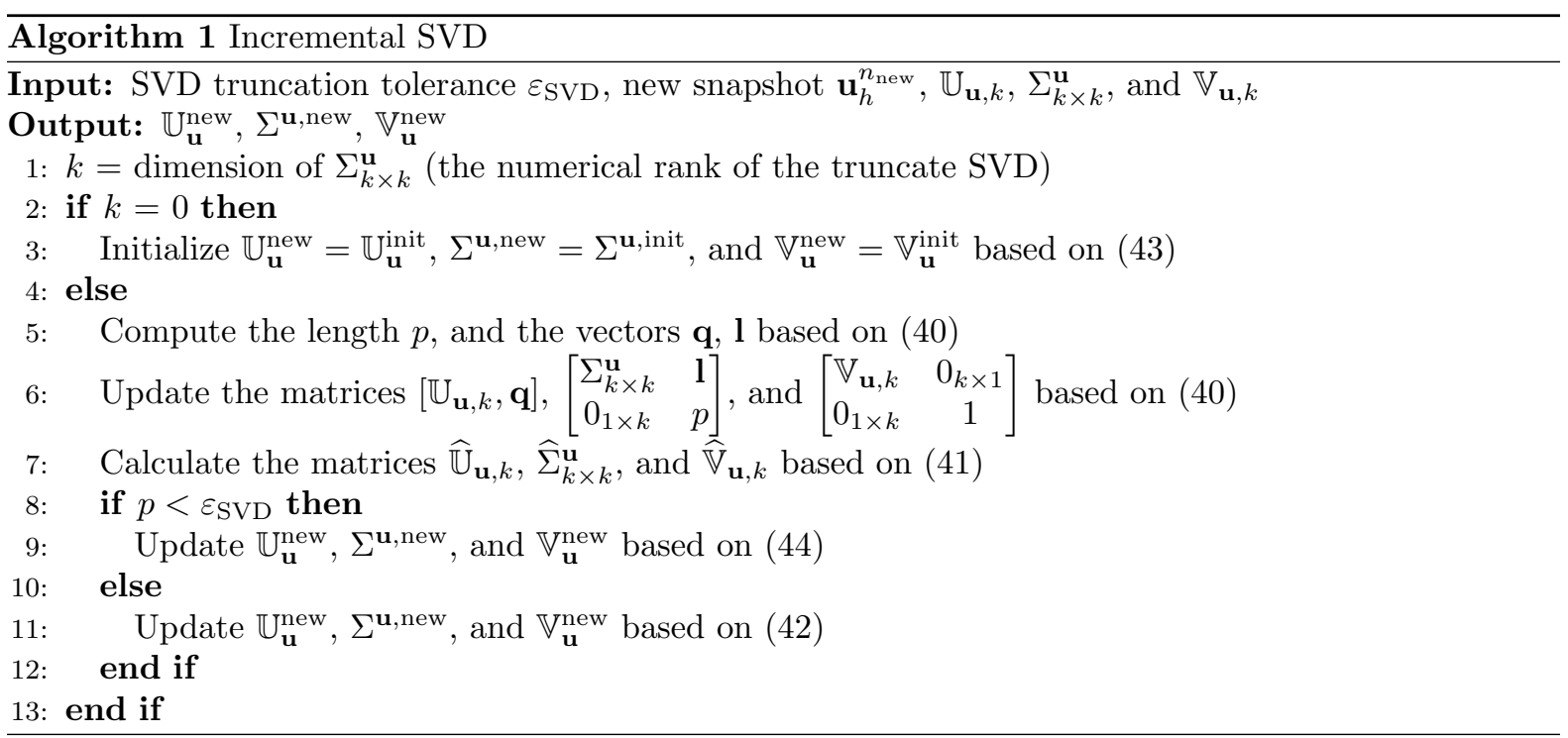

Reamrk 6. In Algorithm 1, the cost of the total truncated SVD takes $\mathcal{O}\left(N \ell k^{2}\right)$ time ${ }^{1}$, and $\mathcal{O}(k(N+\ell+$ $k)$ ) memory. In particular, the truncated $S V D$ is computed in $\mathcal{O}(N \ell k)$ time, and $\mathcal{O}(k(N+\ell))$ memory for low-rank matrices [42], e.g., the POD snapshot matrix $\mathbb{A}_{\mathbf{u}}$ with $k \leq r_{\mathbf{u}} \ll N$. However, a dense SVD would require $\mathcal{O}\left(N \ell^{2}+N^{2} \ell+\ell^{3}\right)$ time, and at least $\mathcal{O}\left(N^{2}+\ell^{2}+N \ell\right)$ [47]. So, the ISVD algorithm is a perfect candidate to save CPU time and memory.

\subsection{Adaptive snapshot selection algorithm}

The adaptive snapshot selection method is proposed to gather snapshots on-the-fly. It determines the time interval $\Delta t_{q}^{\mathbf{u}}(\mathbf{u}=\mathbf{E}, \mathbf{H})$ between snapshots, or the snapshots query time $t_{q}^{\mathbf{u}}(\mathbf{u}=\mathbf{E}, \mathbf{H})$, based on an error control mechanism, in a way similar to adaptive time stepping methods for solving ODEs [48]. The update of the snapshots query time $t_{q}^{\mathrm{u}}$ is based on predicting the growth of the ROM approximation errors $\mathbf{e}_{\mathbf{u}, P}\left(t_{q}^{\mathbf{u}}\right)$ and $\mathbf{e}_{\mathbf{u}, P}\left(t_{q}^{\mathbf{u}}+\Delta t_{q}^{\mathbf{u}}\right)$ because the total errors $\mathbf{e}_{\mathrm{ROM}}(t)\left(t \in\left[0, T_{f}\right]\right)$ and $\mathbf{e}_{\mathrm{ROM}}^{(j)}\left(j=0,1, \cdots, N_{t}\right)$ are only controlled by the errors projected onto the subspace from THEOREMS 3 and 4 , which is spanned by $\Phi_{\mathbf{u}}(\mathbf{u}=\mathbf{E}, \mathbf{H})$. The ROM approximation error $\mathbf{e}_{\mathbf{u}, P}\left(t_{q}^{\mathbf{u}}+\Delta t_{q}^{\mathbf{u}}\right)$ can be estimated by using the first order Taylor series expansion

$$
\mathbf{e}_{\mathbf{u}, P}\left(t_{q}^{\mathbf{u}}+\Delta t_{q}^{\mathbf{u}}\right)=\mathbf{e}_{\mathbf{u}, P}\left(t_{q}^{\mathbf{u}}\right)+\Delta t_{q}^{\mathbf{u}} \mathbf{e}_{\mathbf{u}, P}^{\prime}\left(t_{q}^{\mathbf{u}}\right)+\mathcal{O}\left(\Delta t_{q}^{\mathbf{u}}\right),
$$

with

$$
\mathbf{e}_{\mathbf{u}, P}^{\prime}\left(t_{q}^{\mathbf{u}}\right)=\left[\mathbb{I}-\Psi_{\mathbf{u}}\left(\Psi_{\mathbf{u}}^{T} \mathbb{M} \Psi_{\mathbf{u}}\right)^{-1} \Psi_{\mathbf{u}}^{T} \mathbb{M}\right] \frac{\partial \mathbf{u}_{h}}{\partial t}, \mathbf{u}=\mathbf{E}, \mathbf{H},
$$

\footnotetext{
${ }^{1} \mathbb{Q}$ is a one-column bordered diagonal matrix, so it can be bidiagonalized in $\mathcal{O}\left(k^{2}\right)$ time [1].
} 
where $\frac{\partial \mathbf{u}_{h}}{\partial t}(\mathbf{u}=\mathbf{E}, \mathbf{H})$ has been defined in (8). The optimal step size is then calculated by using the following formulation

$$
\Delta t_{q, \mathrm{opt}}^{\mathbf{u}}=\kappa\left(\frac{\varepsilon_{\text {snapshot }}}{\left\|\mathbf{e}_{\mathbf{u}, P}\left(t_{q}^{\mathbf{u}}+\Delta t_{q}^{\mathbf{u}}\right)\right\|_{\mathbb{M}}}\right)^{\frac{1}{\tau+1}} \cdot \Delta t_{q}^{\mathbf{u}}, \mathbf{u}=\mathbf{E}, \mathbf{H}
$$

where $\kappa$ is a safety factor that is usually set to 0.8 or 0.9 , and $\tau$ is chosen to be either the order of the numerical method or the order of its embedded estimator [1]. Moreover, because $\Delta t_{q}^{\mathbf{u}}$ is not allowed to increase nor to decrease too fast, one can set

$$
\Delta t_{q, \text { new }}^{\mathbf{u}}=\max \left\{\kappa_{\max }, \min \left\{\kappa_{\min }, \kappa\left(\frac{\varepsilon_{\text {snapshot }}}{\left\|\mathbf{e}_{\mathbf{u}, P}\left(t_{q}^{\mathbf{u}}+\Delta t_{q}^{\mathbf{u}}\right)\right\|_{\mathbb{M}}}\right)^{\frac{1}{\tau+1}}\right\}\right\} \cdot \Delta t_{q}^{\mathbf{u}}
$$

where $\kappa_{\max }>1$ and $0<\kappa_{\min }<\kappa$ denote a maximum time step scaling factor and a minimum time step scaling factor, respectively. Besides, according to a standard rule aiming at keeping the error estimate $\left\|\mathbf{e}_{\mathbf{u}, P}\left(t_{q}^{\mathbf{u}}+\Delta t_{q}^{\mathbf{u}}\right)\right\|_{\mathbb{M}}$ close to a snapshot selection error tolerance $\varepsilon_{\text {snapshot }}$, the time interval $\Delta t_{q}^{\mathbf{u}}$ is rejected if $\left\|\mathbf{e}_{\mathbf{u}, P}\left(t_{q}^{\mathbf{u}}+\Delta t_{q}^{\mathbf{u}}\right)\right\|_{\mathbb{M}}>\vartheta \varepsilon_{\text {snapshot }}$ and the time interval $\Delta t_{q}^{\mathbf{u}}$ is chosen as $\Delta t$; otherwise the time interval $\Delta t_{q}^{\mathbf{u}}$ is accepted. Here, $\vartheta$ is an accelerating factor usually taken as 1.2 [49]. The construction of the POD basis is described in Algorithm $2^{2}$.

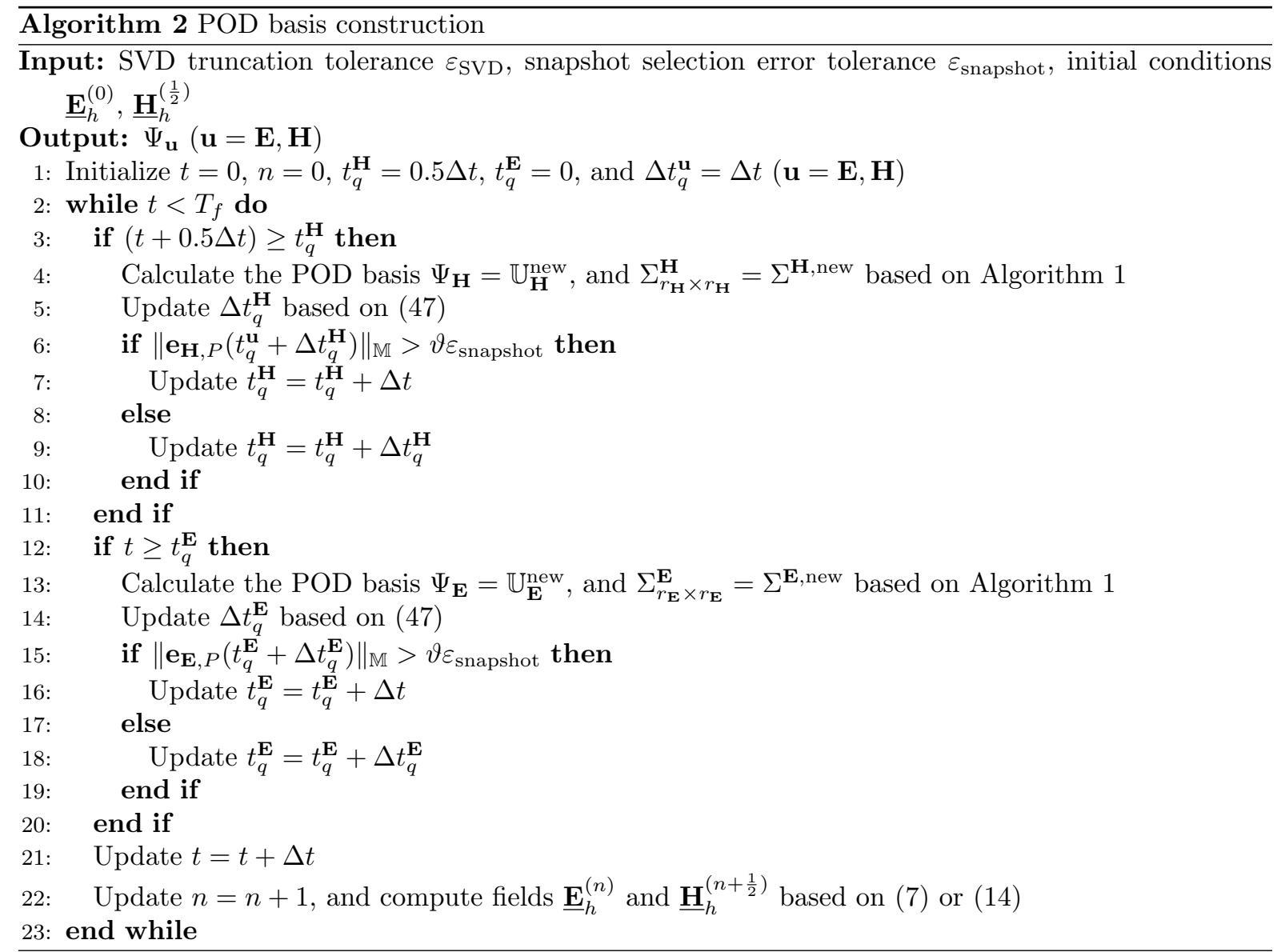

\footnotetext{
${ }^{2}$ The adaptive method can be performed in parallel to electromagnetic simulation without storing snapshots on the disk.
} 


\section{Numerical experiments}

In this section, we present numerical results for the solution of the 2-D time-domain Maxwell equations. More precisely, we consider the case of transverse magnetic (TM) waves

$$
\left\{\begin{array}{l}
\mu_{r} \frac{\partial H_{x}}{\partial t}+\frac{\partial E_{z}}{\partial y}=0 \\
\mu_{r} \frac{\partial H_{y}}{\partial t}-\frac{\partial E_{z}}{\partial x}=0 \\
\varepsilon_{r} \frac{\partial E_{z}}{\partial t}-\frac{\partial H_{y}}{\partial x}+\frac{\partial H_{x}}{\partial y}=0
\end{array}\right.
$$

The DGTD and POD-DGTD methods have been implemented in Matlab 2013b. In order to assess the numerical accuracy of the POD-DGTD method with adaptive snapshot selection (termed the adaptive POD-DGTD method), a POD-DGTD method based on an equispaced snapshot selection algorithm ${ }^{3}$ (termed the equispaced POD-DGTD method) matching the number of snapshots from the adaptive snapshot ROM, is also implemented (see also [28]). All our tests are performed on a computer system equipped a Intel Core i5 $3.3 \mathrm{GHz} \mathrm{CPU}$ and $8 \mathrm{~GB}$ memory. The cost of computing the ROM solution with the equispaced and adaptive snapshot selection algorithms is nearly the same when the number of snapshots of these two strategies is the same, and a detailed CPU time comparison between the DGTD method and the equispaced POD-DGTD method is reported in [28]. So, we do not report CPU time in this work.

\subsection{Standing wave in a PEC square cavity}

In order to verify the stability of the POD-based $\mathrm{ROM}$ with the $\mathrm{LF}_{2}$ time scheme, we first consider the propagation of the $(1,1)$ mode in a PEC square cavity $\Omega_{\square}=\{(x, y) \in[0 \mathrm{~m}, 1 \mathrm{~m}] \times[0 \mathrm{~m}, 1 \mathrm{~m}]\}$ with frequency $f=212 \mathrm{MHz}$ and wavelength $\lambda=1.4 \mathrm{~m}$. The exact solution is given by

$$
\left\{\begin{array}{l}
H_{x}(x, y, t)=-\frac{\pi}{\omega} \sin (\pi x) \cos (\pi y) \sin (\omega t), \\
H_{y}(x, y, t)=\frac{\pi}{\omega} \cos (\pi x) \sin (\pi y) \sin (\omega t) \\
E_{z}(x, y, t)=\sin (\pi x) \sin (\pi y) \cos (\omega t)
\end{array}\right.
$$

where $\omega=2 \pi f$ is the angular frequency. The simulation time is set to 20 periods of the incident plane wave, which corresponds to $28.3 \mathrm{~m}$ (normalized). The simulations are performed using a triangular mesh with 177 nodes and 312 elements, and mesh size $h=1.179 \times 10^{-1} \mathrm{~m}$. The snapshot vectors in this experiment are chosen from the full DGTD solutions, and the adaptive selection method is performed with the error tolerance $\varepsilon_{\text {snapshot }}=10^{-3}$. In the adaptive POD-DGTD method, we set the maximum time step scaling factor $\kappa_{\max }=10$, the minimum time step scaling factor $\kappa_{\min }=0.05$, and the SVD truncation error tolerance is $\varepsilon_{\mathrm{SVD}}=10^{-16}$. These parameters are the same for all our numerical experiments. The time evolution of the global $L^{2}$ error between the exact and the POD-DGTD solutions and the energy defined in (18) are showed in Figure 1, where the $\mathbb{P}_{p}$ approximation denotes the DGTD method of order $p(p=1,2)$.

It is seen that the global $L^{2}$ error stabilizes to a limit value and the energy is exactly conserved, which illustrates the stability of the proposed POD-based ROM under CFL-like condition. In addition, the convergence of the DGTD method has been verified in [28].

\subsection{Plane wave propagation in vacuum}

We then consider a simple problem consisting of the propagation of a plane wave in vacuum to compare the adaptive and equispaced POD-DGTD methods. The computational domain is chosen to be the unit square $\Omega_{\square}=\{(x, y) \in[0 \mathrm{~m}, 1 \mathrm{~m}] \times[0 \mathrm{~m}, 1 \mathrm{~m}]\}$ on which the first order Silver-Müller $\mathrm{ABC}$ is

\footnotetext{
${ }^{3}$ Firstly, one get the POD-based ROM for a snapshot selection error tolerance $\varepsilon_{\text {snapshot }}$. Then, one can calculate the sampling rate $\iota$ matching the dimension of the POD-based ROM. Finally, one can equidistantly extract the $\iota$ transient solutions from the fully DGTD solutions.
} 


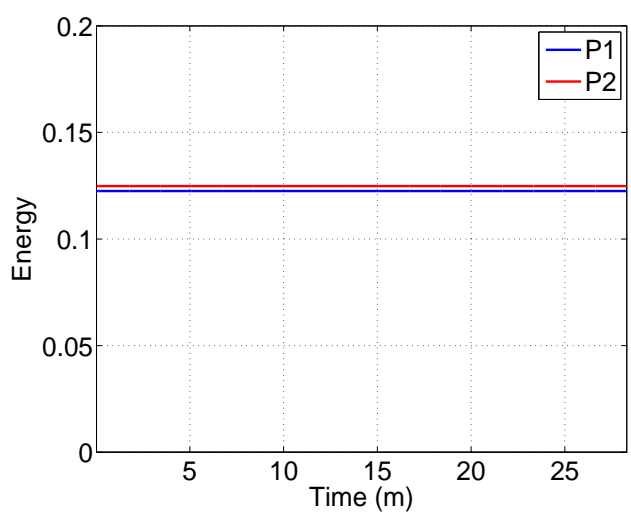

(a)

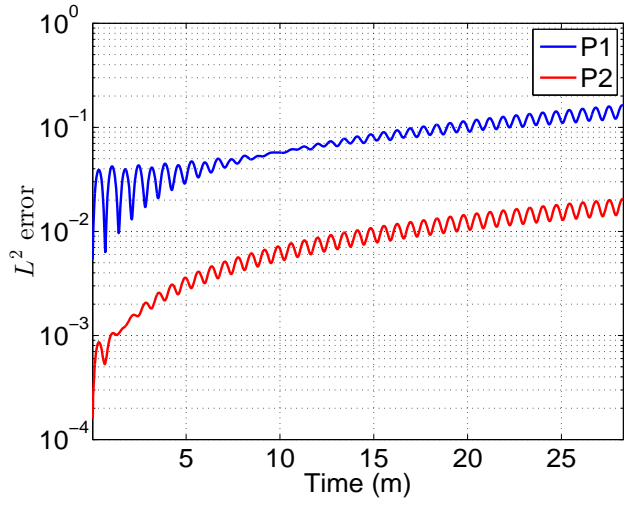

(b)

Figure 1: Standing wave in a PEC square cavity: time evolution of (a) the energy and (b) the global $L^{2}$ error obtained by the POD-DGTD method with $\mathrm{LF}_{2}$ time scheme.

applied. The relative permittivity $\varepsilon_{r}$ and permeability $\mu_{r}$ are set to be 1 everywhere. The incident wave is defined as

$$
\left\{\begin{array}{l}
H_{x}^{\text {inc }}(x, y, t)=0, \\
H_{y}^{\text {inc }}(x, y, t)=-\cos (\omega t-k x), \\
E_{z}^{\text {inc }}(x, y, t)=\cos (\omega t-k x),
\end{array}\right.
$$

where $\omega=2 \pi f$ is the angular frequency with the incident wave frequency $f=300 \mathrm{MHz}$, and $k$ is the wave number. The total simulation time is set to 10 periods of the incident wave oscillation, which corresponds to $10 \mathrm{~m}$ (normalized).

The simulations are performed using a triangular mesh with 2,577 nodes and 4,992 elements, and mesh size $h=2.947 \times 10^{-2} \mathrm{~m}$. A convergence study of the adaptive and equispaced POD-DGTD methods is performed with different snapshot selection error tolerance $\varepsilon_{\text {snapshot }}=10^{-i}(i=0,1, \cdots, 15)$. Figure 2 (a) shows the numerical convergence of the POD-DGTD methods, in which $L^{2}$ error is the global error between the DGTD and POD-DGTD solutions that is integrated in all the elements but not over all the time steps. The presented $L^{2}$ error is the maximum global error over all the time steps. The number of snapshots of these two strategies is the same.

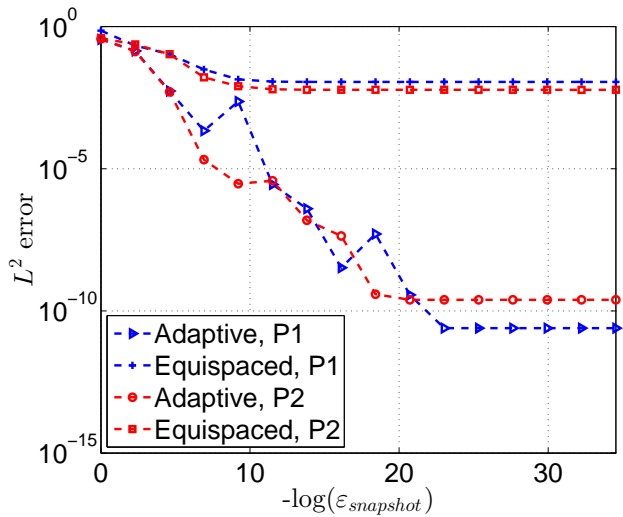

(a)

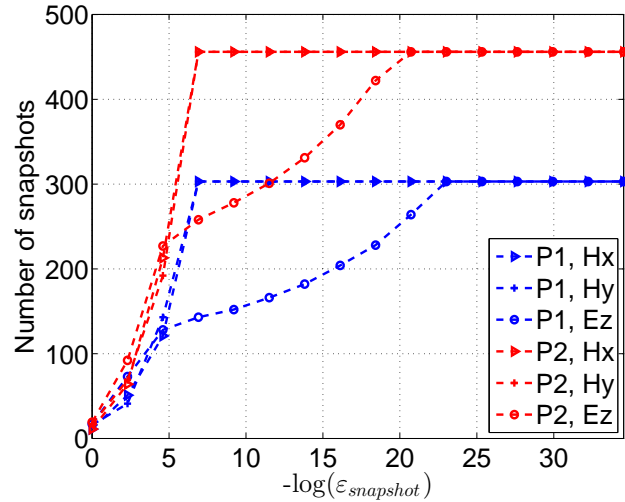

(b)

Figure 2: Plane wave propagation in vacuum: (a) $L^{2}$ error between the DGTD method and the adaptive or equispaced POD-DGTD method. (b) the number of snapshots of the fields $H_{x}, H_{y}$, and $E_{z}$ versus the snapshot selection error tolerance $\varepsilon_{\text {snapshot }}$.

We can observe in Figure 2 (a) that the adaptive POD-DGTD method can achieve higher accuracy compared with the equispaced POD-DGTD method with the same number of snapshots. The number of snapshots of the fields $H_{x}, H_{y}$, and $E_{z}$ with respect to the snapshot selection error tolerance $\varepsilon_{\text {snapshot }}$ 
is shown in Figure 2 (b). Combining Figures 2 (a) and (b), we note that the $L^{2}$ error of the equispaced POD-DGTD method stabilizes to a limit value while the corresponding error of the adaptive PODDGTD method can still decrease when the number of snapshots of the field $E_{z}$ increases. We compare in Figure 3 the time evolution of the electric $E_{z}$ at a selected point with coordinates $(x, y)=(0,1)$ for the exact solution, the DGTD solution, and the adaptive and equispaced POD-DGTD solutions for the snapshot selection error tolerance $\varepsilon_{\text {snapshot }}=10^{-2}$ and $\varepsilon_{\text {snapshot }}=10^{-4}$ with a $\mathbb{P}_{2}$ approximation. A zoom of the solution when $t$ ranges $[6.0,6.2]$ and $[6.5,6.7]$ is also provided in Figure 3. From Figure 3,

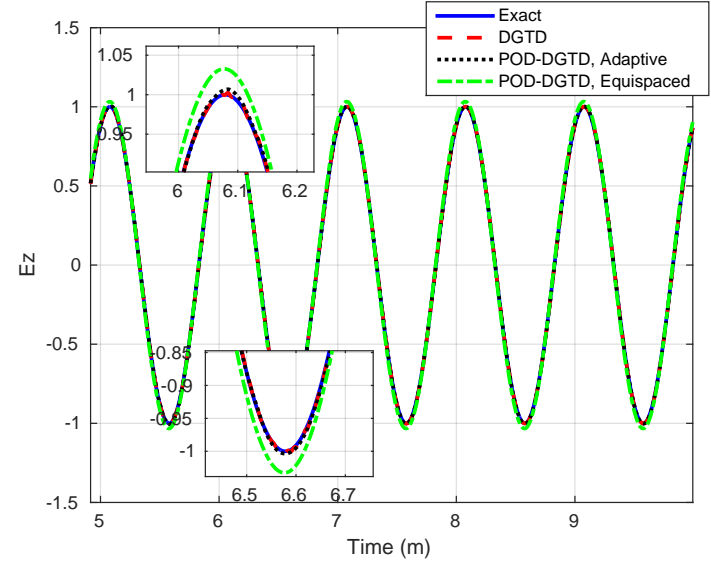

(a)

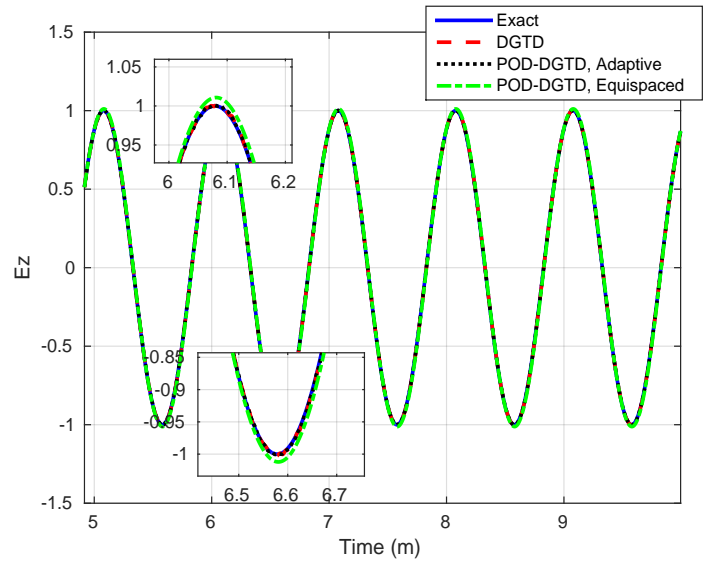

(b)

Figure 3: Plane wave propagation in vacuum: time evolution of the electric $E_{z}$ of the exact solution, the DGTD solution, and the adaptive and equispaced POD-DGTD solutions at a selected point with coordinates $(x, y)=(0,1)$ with the snapshot selection error tolerance (a) $\varepsilon_{\text {snapshot }}=10^{-2}$, (b) $\varepsilon_{\text {snapshot }}=10^{-4}$.

we can find the time evolution of the electric $E_{z}$ obtained by the adaptive or equispaced POD-DGTD

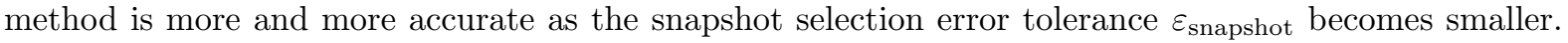
In particular, the solution obtained by the adaptive POD-DGTD method matches the exact and DGTD solutions very well relative to the equispaced POD-DGTD method.

\subsection{Scattering of plane wave by a dielectric disk}

We now consider the problem of the scattering of a plane wave by a dielectric disk. The radius of the disk is $0.6 \mathrm{~m}$, and its relative permittivity and permeability are $\varepsilon_{r, 1}=2.25, \mu_{r, 1}=1$, respectively. The disk is centered at the origin and the computational domain is artificially truncated by the square $\Omega_{\square}=[-1.6 \mathrm{~m}, 1.6 \mathrm{~m}] \times[-1.6 \mathrm{~m}, 1.6 \mathrm{~m}]$. We impose the first order Silver-Müller ABC on the square delimiting the domain. The medium exterior to the dielectric disk is assumed to be vacuum, i.e., $\varepsilon_{r, 2}=1$, $\mu_{r, 2}=1$. The incident wave is defined in (50). The incident wave frequency, $f$, is set to $f=300 \mathrm{MHz}$.

The simulations are performed using a triangular mesh with 2,873 nodes, 5,568 elements of which 512 elements are located inside the dielectric disk, and mesh size $h=9.9 \times 10^{-2} \mathrm{~m}$. The total simulation time is set to 10 periods of the incident wave oscillation. The numerical convergence of the adaptive and equispaced POD-DGTD methods with $\mathbb{P}_{1}$ and $\mathbb{P}_{2}$ approximation is displayed in Figure 4 . We can make similar observations from Figure 4 as from Figure 2. The time evolution of the fields $H_{y}$ and $E_{z}$ at a given point, which are obtained by the DGTD method, and the adaptive and equispaced POD-DGTD methods for the snapshot selection error tolerance $\varepsilon_{\text {snapshot }}=10^{-2}$, are shown in Figure 5 . We observe from Figure 5 that the time evolution of the $H_{y}$ and $E_{z}$ components obtained by the adaptive PODDGTD method are in good agreement with that of the DGTD method, while the equispaced POD-DGTD solution is not accurate enough. The distributions of the real part of $E_{z}$ in the Fourier domain during the last period of oscillation of the incident wave with a $\mathbb{P}_{2}$ approximation and the corresponding field based on the adaptive and equispaced snapshot selection algorithms are shown when $\varepsilon_{\text {snapshot }}=10^{-6}$ in Figure 6 .

Finally, we show how the POD-DGTD methods can be potentially applied in real applications. Since we already have the POD basis and the ROM for $\varepsilon_{r, 1}^{\text {ref }}=2.25$, now we can use them instead of the full DGTD formulations to compute the electromagnetic fields when $\varepsilon_{r, 1}$ ranges $\left[\varepsilon_{r, 1}^{\min }, \varepsilon_{r, 1}^{\max }\right]=[1.5,4]$. The 


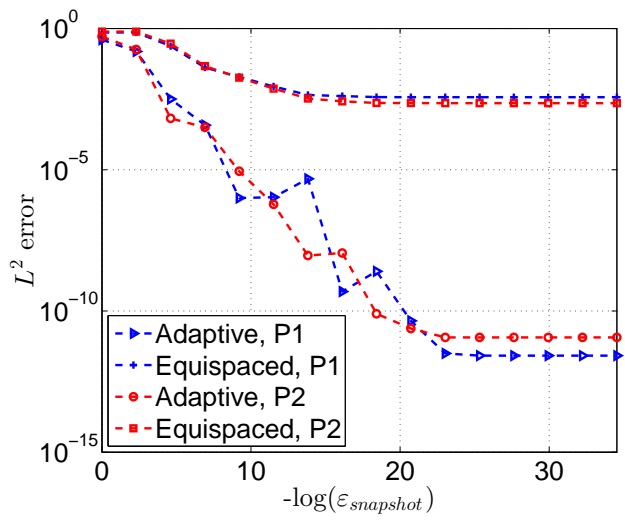

(a)

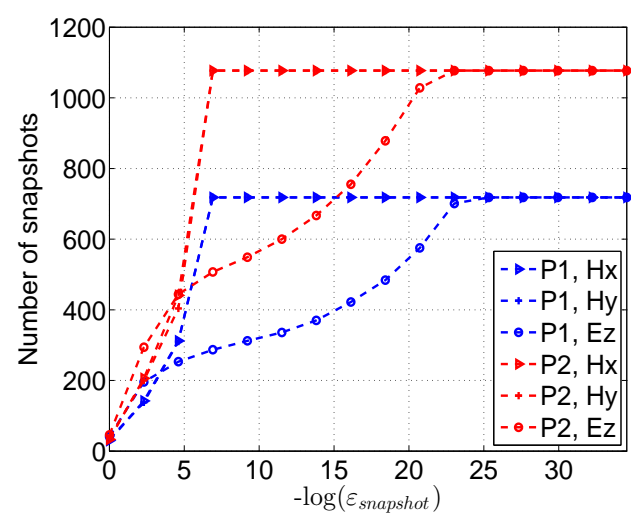

(b)

Figure 4: Scattering of plane wave by a dielectric disk: (a) $L^{2}$ errors for the adaptive and equispaced POD-DGTD methods. (b) the number of snapshots of the fields $H_{x}, H_{y}$, and $E_{z}$ versus the snapshot selection error tolerance $\varepsilon_{\text {snapshot }}$.
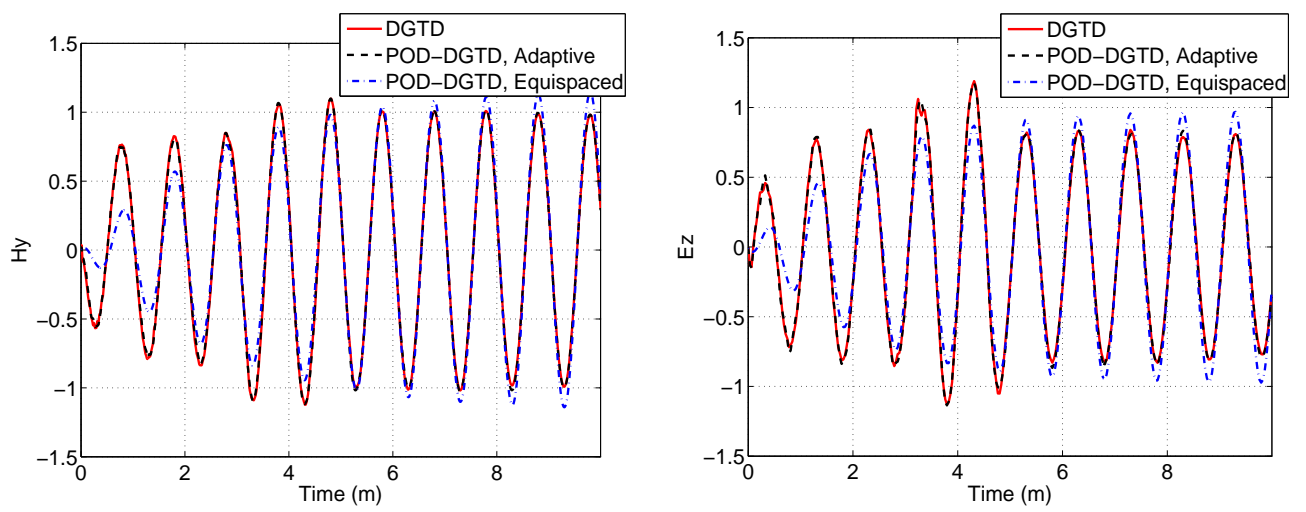

Figure 5: Scattering of plane wave by a dielectric disk: the time evolution of the fields $H_{y}$ (left) and $E_{z}$ (right) at a given point obtained by the DGTD method, and the adaptive and equispaced POD-DGTD methods for the snapshot selection error tolerance $\varepsilon_{\text {snapshot }}=10^{-2}$.

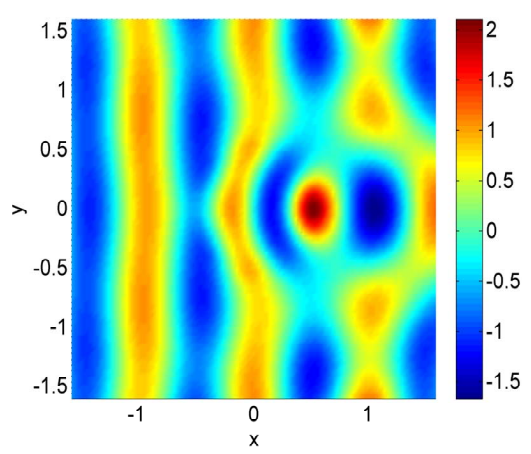

(a)

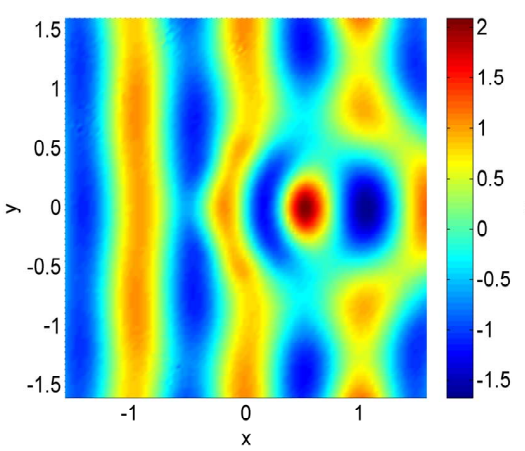

(b)

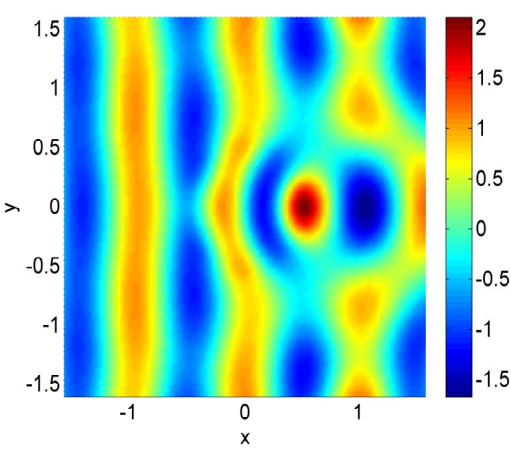

(c)

Figure 6: Scattering of plane wave by a dielectric disk: contour lines of the real part of the $E_{z}$ component in the Fourier domain. (a) solution from the DGTD method. (b) the solution from the adaptive POD-DGTD method. (c) the solution from the equispaced POD-DGTD method. 

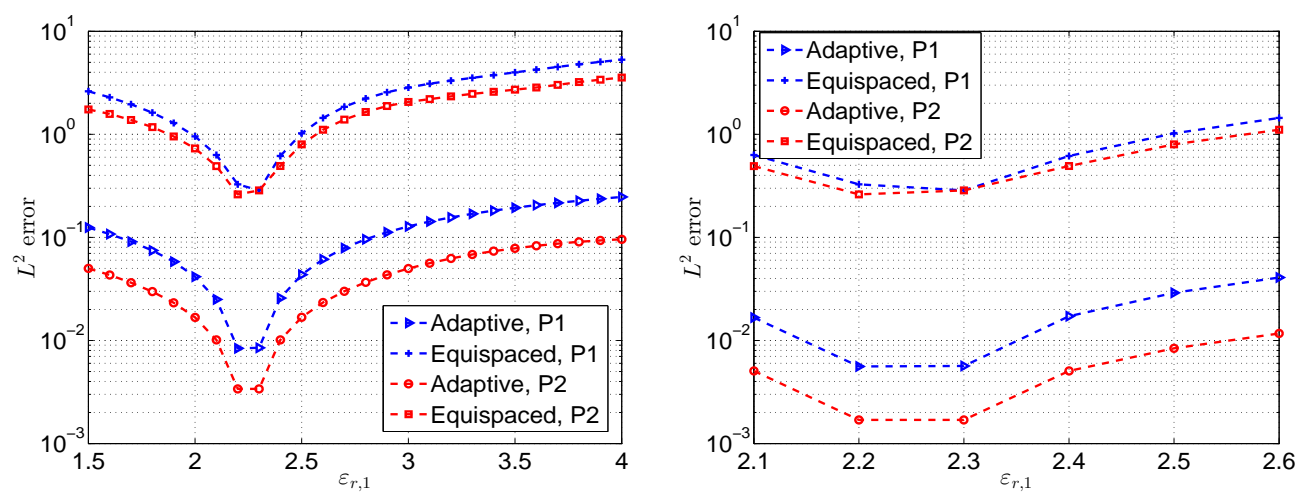

Figure 7: Scattering of plane wave by a dielectric disk: performance of the POD-DGTD methods (left), zoom (right), for the snapshot selection error tolerance $\varepsilon_{\text {snapshot }}=10^{-2}$.

error is presented in Figure 7. We can find from Figure 7 that the POD-DGTD method is applicable in electromagnetic computations with varying parameters. The adaptive snapshot selection strategy proposed in this paper performs much better that the equispaced snapshot selection strategy. In addition, we can see that the error of the adaptive POD-DGTD method is also sensitive to the difference between the underlying parameters and reference parameters. We will consider the parametrized time-dependent problems in the near future. Some related contributions please refer to $[26,50,51]$.

\subsection{Scattering of a modulated Gaussian by an airfoil profile}

We finally consider the problem of the scattering of a modulated Gaussian by an airfoil profile, which is a cross-section of a three-dimensional wing of an airplane. The computational domain is chosen to be the rectangle $\Omega=[-1 \mathrm{~m}, 2 \mathrm{~m}] \times[-1 \mathrm{~m}, 1 \mathrm{~m}]$. We impose the first order Silver-Müller ABC on the rectangle delimiting the domain, while the surface of the airfoil is assumed to be the PEC condition. In the present case, the wave is considered to be emitted from a localized source modeled as

$$
J_{z}^{s}(\vec{x}, t)=A e^{-\left(\frac{t-4 \tau}{\tau}\right)^{2}} \sin \left(2 \pi f_{c}(t-4 \tau)\right) \frac{g(\vec{x})}{\|g(\vec{x})\|},
$$

where $A$ is the amplitude of the signal, $\tau=1.7 \times 10^{3} \mathrm{ps}, f_{c}=1.2 \mathrm{GHz}$, and $g(\vec{x})$ is a two-dimensional Gaussian function with $\left(x_{0}, y_{0}\right)=(-0.3,0.0)$ the center of the Gaussian spatial support

$$
g(\vec{x})=e^{-\beta\left(\left(x-x_{0}\right)^{2}+\left(y-y_{0}\right)^{2}\right)} .
$$

Here, the parameter $\beta$ has been chosen such that the source term $J_{z}^{s}$ is strongly localized. In this study, we set $\beta=2.5 \times 10^{3}$. The total simulation time, $T_{f}$, is set to $T_{f}=10 \mathrm{~m}$ (normalized). Note that there is no analytical solution for this case.

The simulations are performed using a mesh with 8,436 nodes and 16,460 elements, and mesh size $h=3.993 \times 10^{-2} \mathrm{~m}$. The result of a numerical convergence study of the adaptive and equispaced PODDGTD methods with $\mathbb{P}_{1}$ and $\mathbb{P}_{2}$ approximation is shown in Figure 8. We can make similar observations from Figure 8 as from Figures 2 and 4 . The time evolution of the fields $H_{y}$ and $E_{z}$ at a selected point of the mesh for the DGTD method, and the adaptive and equispaced POD-DGTD methods when $\varepsilon_{\text {snapshot }}=10^{-6}$ with a $\mathbb{P}_{2}$ approximation are shown in Figure 9. The intensity of the electric field of the DGTD method and the adaptive and equispaced POD-DGTD methods when $\varepsilon_{\text {snapshot }}=10^{-6}$ with a $\mathbb{P}_{2}$ approximation are presented in Figure 10.

\section{Conclusions and outlook}

We have proposed and studied a POD-based ROM in combination with a high order DGTD method for the generation of snapshots, in the context of the numerical solution of the system of time-domain Maxwell equations modeling electromagnetic wave propagation in homogeneous media and heterogeneous media as well. The DGTD method considered here makes use of a centered scheme for the definition 


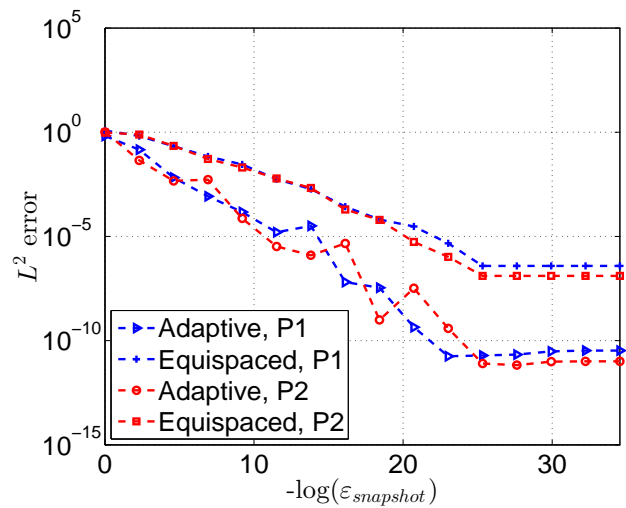

(a)

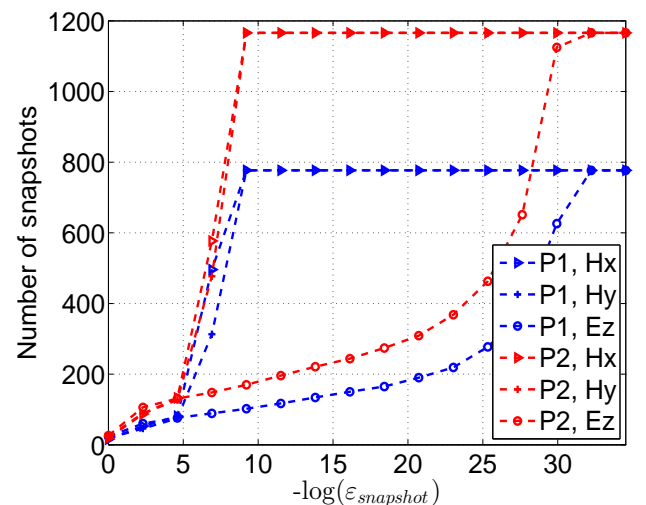

(b)

Figure 8: Scattering of a modulated Gaussian by an airfoil profile: (a) $L^{2}$ error for the adaptive and equispaced POD-DGTD method. (b) number of snapshots of the fields $H_{x}, H_{y}$, and $E_{z}$ versus the snapshot selection error tolerance $\varepsilon_{\text {snapshot }}$.

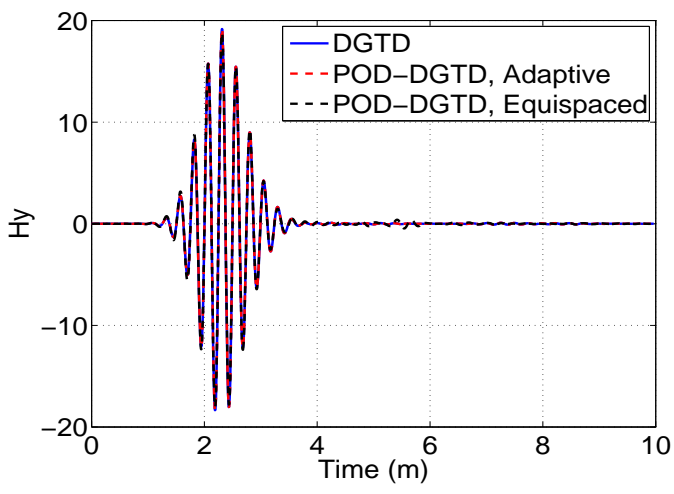

(a)

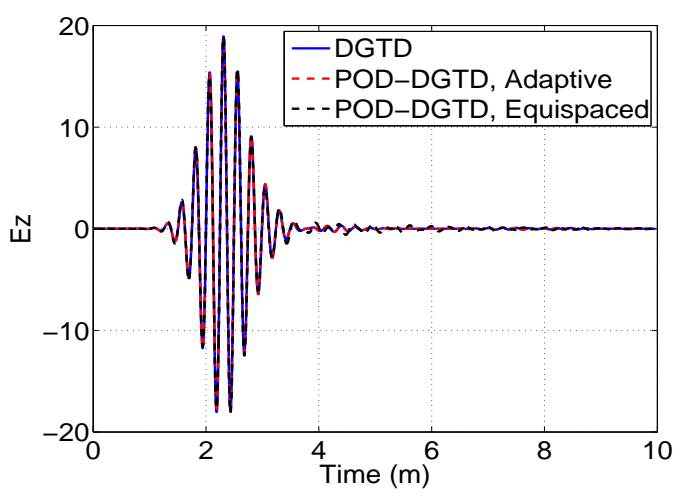

(b)

Figure 9: Scattering of a modulated Gaussian by an airfoil profile: time evolution of the fields $H_{y}$ (left) and $E_{z}$ (right), at point $(x, y)=(-0.541,0)$, for the DGTD and POD-DGTD methods with a $\mathbb{P}_{2}$ approximation.

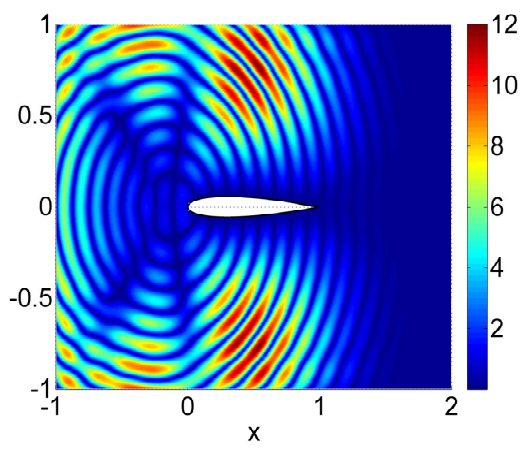

(a)

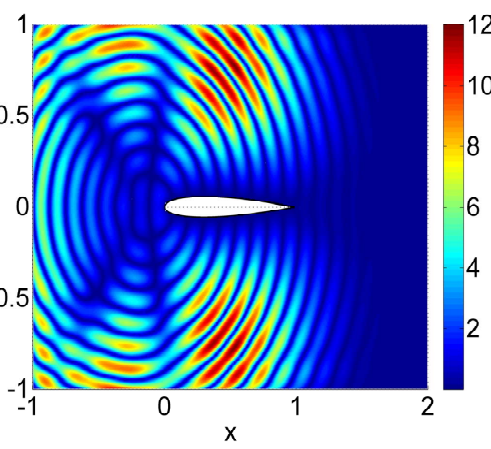

(b)

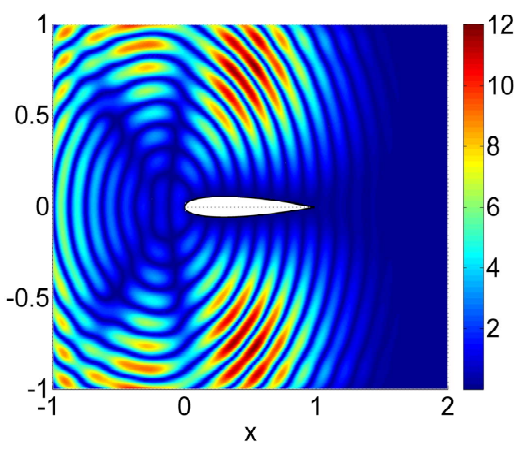

(c)

Figure 10: Scattering of a modulated Gaussian by an airfoil profile: time evolution of the intensity of the electric filed, $\left|E_{z}\right|$, at time $\mathrm{t}=3.20 \mathrm{~m}$. (a) the solution of the DGTD method. (b) the solution of the adaptive POD-DGTD method. (c) the solution of the equispaced POD-DGTD method. 
of the numerical traces of the electric and magnetic fields at element interfaces, with the $\mathrm{LF}_{2}$ scheme for the time integration of the associated semi-discrete equations. The same scheme has been applied for the time integration of the semi-discrete ROM equations. However, other options could be adopted such as the implicit Crank-Nicolson scheme. This possibility of using a time integration scheme for the POD-projected semi-discrete system, which is different from the time scheme adopted in the time-domain method used for generating the snapshots, will be the subject of a future study with the goal of further improving the overall computational efficiency of the POD-DGTD method.

We proved that the POD-based ROM conserves the discrete electromagnetic energy and it is stable under some CFL-like stability condition. Error bounds for the proposed POD-based ROM method have been derived at both the semi-discrete and fully discrete level, which are direct extensions of previously published results for the wave equation [3]. In the same spirit, by extending the approach described in [1], an adaptive snapshot selection algorithm has been designed, which leverages the error bounds. In particular, we introduced the weight $\mathbb{M}$ to measure the control error and changed the standard snapshot selection rule to a strategy aiming at keeping the error estimate $\left\|\mathbf{e}_{\mathbf{u}, P}\left(t_{q}^{\mathbf{u}}+\Delta t_{q}^{\mathbf{u}}\right)\right\|_{\mathbb{M}}$ close to the snapshot selection error tolerance $\varepsilon_{\text {snapshot }}$ in the adaptive method. An ISVD algorithm has been exploited to update the SVD on-the-fly when a new snapshot is added. Numerical results on 2-D test problems indicate that the proposed methodology is effective for electromagnetic computations. Based on these results, we will consider more complex 3-D realistic applications in the near future. Moreover, several further developments and applications of this POD-DGTD method can be considered among which Uncertainty Quantification (UQ) is included. Regarding this topic, we plan to investigate multifidelity UQ methods [33], which leverage low-fidelity models to obtain computational speedups in solving UQ tasks.

\section{Acknowledgments}

This work is supported by NSFC (Grant No. 61772003) and Science Strength Promotion Programme of UESTC.

\section{Appendix A. Proof of Theorem 3}

Differentiating $\mathbf{e}_{\mathbf{E}, V}(t)$ with respect to time, we get

$$
\begin{aligned}
\mathbf{e}_{\mathbf{E}, V}^{\prime}(t) & =\Psi_{\mathbf{E}}\left[\left(\Psi_{\mathbf{E}}^{T} \mathbb{M} \Psi_{\mathbf{E}}\right)^{-1} \Psi_{\mathbf{E}}^{T} \mathbb{M} \frac{\partial \mathbf{E}_{h}}{\partial t}-\alpha_{\mathbf{E}}^{\prime}(t)\right] \\
& =\Psi_{\mathbf{E}}\left\{\varepsilon_{r}^{-1}\left(\Psi_{\mathbf{E}}^{T} \mathbb{M} \Psi_{\mathbf{E}}\right)^{-1} \Psi_{\mathbf{E}}^{T}\left[\left(\mathbb{K}-\mathbb{S}^{i}\right) \underline{\mathbf{H}}_{h}-\mathbb{S}^{h} \underline{\widehat{\mathbf{H}}}_{h}-\mathbf{B}^{h}(t)\right]\right. \\
& \left.-\varepsilon_{r}^{-1}\left(\Psi_{\mathbf{E}}^{T} \mathbb{M} \Psi_{\mathbf{E}}\right)^{-1} \Psi_{\mathbf{E}}^{T}\left[\left(\mathbb{K}-\mathbb{S}^{i}\right) \Psi_{\mathbf{H}} \alpha_{\mathbf{H}}(t)-\mathbb{S}^{h} \widehat{\Psi}_{\mathbf{H}} \widehat{\alpha}_{\mathbf{H}}(t)-\mathbf{B}^{h}(t)\right]\right\} \\
& =\varepsilon_{r}^{-1} \Psi_{\mathbf{E}}\left(\Psi_{\mathbf{E}}^{T} \mathbb{M} \Psi_{\mathbf{E}}\right)^{-1} \Psi_{\mathbf{E}}^{T}\left[\left(\mathbb{K}-\mathbb{S}^{i}\right)\left(\underline{\mathbf{H}}_{h}-\Psi_{\mathbf{H}} \alpha_{\mathbf{H}}(t)\right)-\mathbb{S}^{h}\left(\widehat{\hat{\mathbf{H}}}_{h}-\widehat{\Psi}_{\mathbf{H}} \widehat{\alpha}_{\mathbf{H}}(t)\right)\right] .
\end{aligned}
$$

According to the definitions of (8) and (25), the $\mathbf{e}_{\mathbf{E}, V}(t)$ satisfies the equations

$$
\mathbf{e}_{\mathbf{E}, V}^{\prime}(t)=\left\{\begin{array}{l}
\varepsilon_{r}^{-1} \Psi_{\mathbf{E}}\left(\Psi_{\mathbf{E}}^{T} \mathbb{M} \Psi_{\mathbf{E}}\right)^{-1} \Psi_{\mathbf{E}}^{T}\left(\mathbb{K}-\mathbb{S}^{i}-\mathbb{S}^{h}\right) \mathbf{e}_{\mathbf{H}}(t), \text { if } F_{h}^{B}=\Gamma_{m}, \\
\varepsilon_{r}^{-1} \Psi_{\mathbf{E}}\left(\Psi_{\mathbf{E}}^{T} \mathbb{M} \Psi_{\mathbf{E}}\right)^{-1} \Psi_{\mathbf{E}}^{T}\left[\left(\mathbb{K}-\mathbb{S}^{i}\right) \mathbf{e}_{\mathbf{H}}(t)-\mathbb{S}^{h} \mathbf{e}_{\mathbf{E}}(t)\right], \text { if } F_{h}^{B}=\Gamma_{a} .
\end{array}\right.
$$

Similarly, the $\mathbf{e}_{\mathbf{H}, V}(t)$ satisfies the equations

$$
\mathbf{e}_{\mathbf{H}, V}^{\prime}(t)=\left\{\begin{array}{l}
\mu_{r}^{-1} \Psi_{\mathbf{H}}\left(\Psi_{\mathbf{H}}^{T} \mathbb{M} \Psi_{\mathbf{H}}\right)^{-1} \Psi_{\mathbf{H}}^{T}\left(-\mathbb{K}+\mathbb{S}^{i}+\mathbb{S}^{e}\right) \mathbf{e}_{\mathbf{E}}(t), \text { if } F_{h}^{B}=\Gamma_{m}, \\
\mu_{r}^{-1} \Psi_{\mathbf{H}}\left(\Psi_{\mathbf{H}}^{T} \mathbb{M} \Psi_{\mathbf{H}}\right)^{-1} \Psi_{\mathbf{H}}^{T}\left[\left(-\mathbb{K}+\mathbb{S}^{i}\right) \mathbf{e}_{\mathbf{E}}(t)+\mathbb{S}^{e} \mathbf{e}_{\mathbf{H}}(t)\right], \text { if } F_{h}^{B}=\Gamma_{a}
\end{array}\right.
$$

Based on (13) and (26), we have

$$
\mathbf{e}_{\mathbf{E}, V}(0)=0, \quad \mathbf{e}_{\mathbf{H}, V}(0)=0 .
$$


We first consider the situation with $F_{h}^{B}=\Gamma_{m}$. Substituting (25) into the first relations of (A.2) and (A.3), we get

$$
\left\{\begin{array}{l}
\mathbf{e}_{\mathbf{E}, V}^{\prime}(t)=\varepsilon_{r}^{-1} \Psi_{\mathbf{E}}\left(\Psi_{\mathbf{E}}^{T} \mathbb{M} \Psi_{\mathbf{E}}\right)^{-1} \Psi_{\mathbf{E}}^{T}\left(\mathbb{K}-\mathbb{S}^{i}-\mathbb{S}^{h}\right)\left(\mathbf{e}_{\mathbf{H}, P}(t)+\mathbf{e}_{\mathbf{H}, V}(t)\right), \\
\mathbf{e}_{\mathbf{H}, V}^{\prime}(t)=\mu_{r}^{-1} \Psi_{\mathbf{H}}\left(\Psi_{\mathbf{H}}^{T} \mathbb{M} \Psi_{\mathbf{H}}\right)^{-1} \Psi_{\mathbf{H}}^{T}\left(-\mathbb{K}+\mathbb{S}^{i}+\mathbb{S}^{e}\right)\left(\mathbf{e}_{\mathbf{E}, P}(t)+\mathbf{e}_{\mathbf{E}, V}(t)\right) .
\end{array}\right.
$$

According to (A.4) and (A.5), we can easily get the explicit formula of $\mathbf{e}_{\mathbf{E}, V}(t)$ and $\mathbf{e}_{\mathbf{H}, V}(t)$ based the theory of nonhomogeneous and linear differential equations

$$
\Pi(t)=\int_{0}^{t} \mathrm{e}^{\left[\widehat{\Psi}\left(\widehat{\Psi}^{T} \widehat{\mathbb{M}} \widehat{\Psi}\right)^{-1} \widehat{\Psi}^{T} \mathbb{R}(t-\tau)\right]} \cdot\left\{\widehat{\Psi}\left(\widehat{\Psi}^{T} \widehat{\mathbb{M}} \widehat{\Psi}\right)^{-1} \widehat{\Psi}^{T} \mathbb{R}\left[\begin{array}{l}
\mathbf{e}_{\mathbf{E}, P}(t) \\
\mathbf{e}_{\mathbf{H}, P}(t)
\end{array}\right]\right\} d \tau
$$

where $\Pi(t)=\left[\begin{array}{l}\mathbf{e}_{\mathbf{E}, V}(t) \\ \mathbf{e}_{\mathbf{H}, V}(t)\end{array}\right]$. Based on the properties of the matrix exponential, (28), and (29), we have

$$
\begin{aligned}
\Pi(t) & =\widehat{\Psi} \int_{0}^{t} \mathrm{e}^{\left[\left(\widehat{\Psi}^{T} \widehat{\mathbb{M}} \widehat{\Psi}\right)^{-1} \widehat{\Psi}^{T} \mathbb{R} \widehat{\Psi}(t-\tau)\right]} \cdot\left\{\left(\widehat{\Psi}^{T} \widehat{\mathbb{M}} \widehat{\Psi}\right)^{-1} \widehat{\Psi}^{T} \mathbb{R}\left[\begin{array}{l}
\mathbf{e}_{\mathbf{E}, P}(t) \\
\mathbf{e}_{\mathbf{H}, P}(t)
\end{array}\right]\right\} d \tau \\
& =\widehat{\Psi} \int_{0}^{t} \mathrm{e}^{\left[\left(\widehat{\Psi}^{T} \widehat{\mathbb{M}} \widehat{\Psi}\right)^{-1} \widehat{\Psi}^{T} \mathbb{R} \widehat{\Psi}(t-\tau)\right]} \cdot\left\{\left(\widehat{\Psi}^{T} \widehat{\mathbb{M}} \widehat{\Psi}\right)^{-1} \widehat{\Psi}^{T} \mathbb{R} \widehat{\Phi}\left(\widehat{\Phi}^{T} \widehat{\mathbb{M}} \widehat{\Phi}\right)^{-1} \widehat{\Phi}^{T} \widehat{\mathbb{M}}^{\frac{1}{2}}\right\}\left\{\widehat{\mathbb{M}}^{\frac{1}{2}}\left[\begin{array}{l}
\mathbf{e}_{\mathbf{E}, P}(t) \\
\mathbf{e}_{\mathbf{H}, P}(t)
\end{array}\right]\right\} d \tau \\
& =\widehat{\Psi} F\left(T_{f} ;\left(\widehat{\Psi} \widehat{\Psi}^{T} \widehat{\mathbb{M}} \widehat{\Psi}\right)^{-1} \widehat{\Psi}^{T} \mathbb{R} \widehat{\Psi}\right)\left[\left(\widehat{\Psi}^{T} \widehat{\mathbb{M}} \widehat{\Psi}\right)^{-1} \widehat{\Psi}^{T} \mathbb{R} \widehat{\Phi}\left(\widehat{\Phi}^{T} \widehat{\mathbb{M}} \widehat{\Phi}\right)^{-1} \widehat{\Phi}^{T} \widehat{\mathbb{M}} \frac{1}{2}\right]\left(\widehat{\mathbb{M}} \widehat{1}^{\frac{1}{2}}\left[\begin{array}{l}
\mathbf{e}_{\mathbf{E}, P}(t) \\
\mathbf{e}_{\mathbf{H}, P}(t)
\end{array}\right]\right) .
\end{aligned}
$$

One can easily get the following inequality based on (27), (30), and (A.7)

$$
\begin{aligned}
\int_{0}^{T_{f}}\|\Pi(t)\|_{\widehat{\mathbb{M}}}^{2} d t & =\int_{0}^{T_{f}}\left\|\mathbf{e}_{\mathbf{E}, V}(t)\right\|_{\mathbb{M}}^{2} d t+\int_{0}^{T_{f}}\left\|\mathbf{e}_{\mathbf{H}, V}(t)\right\|_{\mathbb{M}}^{2} d t \\
& \leq\left(\left\|F\left(T_{f} ;\left(\widehat{\Psi}^{T} \widehat{\mathbb{M}} \widehat{\Psi}\right)^{-1} \widehat{\Psi}^{T} \mathbb{R} \widehat{\Psi}\right)\right\|_{\widehat{\Psi} \widehat{\mathbb{M}}_{\widehat{\Psi}}}^{2}\left\|\widehat{\Psi}^{T} \mathbb{R} \widehat{\Phi}\left(\widehat{\Phi}^{T} \widehat{\mathbb{M}} \widehat{\Phi}\right)^{-1} \widehat{\Phi}^{T} \widehat{\mathbb{M}}^{\frac{1}{2}}\right\|_{(\widehat{\Psi} T \widehat{\mathbb{M}} \widehat{\Psi})^{-1}}^{2}\right) \\
& \times\left(\int_{0}^{T_{f}}\left\|\mathbf{e}_{\mathbf{E}, P}(t)\right\|_{\mathbb{M}}^{2} d t+\int_{0}^{T_{f}}\left\|\mathbf{e}_{\mathbf{H}, P}(t)\right\|_{\mathbb{M}}^{2} d t\right) .
\end{aligned}
$$

The total error $\mathbf{e}_{\mathrm{ROM}}(t)$ satisfies

$$
\int_{0}^{T_{f}}\left\|\mathbf{e}_{\mathrm{ROM}}(t)\right\|_{\mathbb{M}}^{2} d t=\int_{0}^{T_{f}}\left\|\mathbf{e}_{\mathbf{E}}(t)\right\|_{\mathbb{M}}^{2} d t+\int_{0}^{T_{f}}\left\|\mathbf{e}_{\mathbf{H}}(t)\right\|_{\mathbb{M}}^{2} d t .
$$

Because $\mathbf{e}_{\mathbf{u}, P}(t)$ and $\mathbf{e}_{\mathbf{u}, V}(t)(\mathbf{u}=\mathbf{E}, \mathbf{H})$ belong to $\operatorname{span} \Phi_{\mathbf{u}}$ and $\operatorname{span} \Psi_{\mathbf{u}},($ A.9) by using the Pythagorean theorem then becomes

$$
\begin{aligned}
\int_{0}^{T_{f}}\left\|\mathbf{e}_{\mathrm{ROM}}(t)\right\|_{\mathbb{M}}^{2} d t & =\left(\int_{0}^{T_{f}}\left\|\mathbf{e}_{\mathbf{E}, P}(t)\right\|_{\mathbb{M}}^{2} d t+\int_{0}^{T_{f}}\left\|\mathbf{e}_{\mathbf{H}, P}(t)\right\|_{\mathbb{M}}^{2} d t\right) \\
& +\left(\int_{0}^{T_{f}}\left\|\mathbf{e}_{\mathbf{E}, V}(t)\right\|_{\mathbb{M}}^{2} d t+\int_{0}^{T_{f}}\left\|\mathbf{e}_{\mathbf{H}, V}(t)\right\|_{\mathbb{M}}^{2} d t\right) .
\end{aligned}
$$

Theorem 4 is proved by substituting (A.8) into (A.10). If $F_{h}^{B}=\Gamma_{a}$, one can get a similar conclusion.

\section{Appendix B. Proof of Theorem 4}

Analogously to (A.2) and (A.3), we can obtain

$$
\left\{\begin{array}{l}
\frac{\mathbf{e}_{\mathbf{E}, V}^{(n+1)}-\mathbf{e}_{\mathbf{E}, V}^{(n)}}{\Delta t}=\varepsilon_{r}^{-1} \Psi_{\mathbf{E}}\left(\Psi_{\mathbf{E}}^{T} \mathbb{M} \Psi_{\mathbf{E}}\right)^{-1} \Psi_{\mathbf{E}}^{T}\left(\mathbb{K}-\mathbb{S}^{i}-\mathbb{S}^{h}\right)\left(\mathbf{e}_{\mathbf{H}, P}^{\left(n+\frac{1}{2}\right)}+\mathbf{e}_{\mathbf{H}, V}^{\left(n+\frac{1}{2}\right)}\right), \\
\frac{\mathbf{e}_{\mathbf{H}, V}^{\left(n+\frac{3}{2}\right)}-\mathbf{e}_{\mathbf{H}, V}^{\left(n+\frac{1}{2}\right)}}{\Delta t}=\mu_{r}^{-1} \Psi_{\mathbf{H}}\left(\Psi_{\mathbf{H}}^{T} \mathbb{M} \Psi_{\mathbf{H}}\right)^{-1} \Psi_{\mathbf{H}}^{T}\left(-\mathbb{K}+\mathbb{S}^{i}+\mathbb{S}^{e}\right)\left(\mathbf{e}_{\mathbf{E}, P}^{(n+1)}+\mathbf{e}_{\mathbf{E}, V}^{(n+1)}\right),
\end{array}\right.
$$


for $n=0,1, \cdots, N_{t}-1$ when $F_{h}^{B}=\Gamma_{m}$, and

$$
\left\{\begin{aligned}
\frac{\mathbf{e}_{\mathbf{E}, V}^{(n+1)}-\mathbf{e}_{\mathbf{E}, V}^{(n)}}{\Delta t} & =\varepsilon_{r}^{-1} \Psi_{\mathbf{E}}\left(\Psi_{\mathbf{E}}^{T} \mathbb{M} \Psi_{\mathbf{E}}\right)^{-1} \Psi_{\mathbf{E}}^{T}\left[\left(\mathbb{K}-\mathbb{S}^{i}\right)\left(\mathbf{e}_{\mathbf{H}, P}^{\left(n+\frac{1}{2}\right)}+\mathbf{e}_{\mathbf{H}, V}^{\left(n+\frac{1}{2}\right)}\right)\right. \\
& \left.-\mathbb{S}^{h}\left(\mathbf{e}_{\mathbf{E}, P}^{(n)}+\mathbf{e}_{\mathbf{E}, V}^{(n)}\right)\right], \\
\frac{\mathbf{e}_{\mathbf{H}, V}^{\left(n+\frac{3}{2}\right)}-\mathbf{e}_{\mathbf{H}, V}^{\left(n+\frac{1}{2}\right)}}{\Delta t} & =\mu_{r}^{-1} \Psi_{\mathbf{H}}\left(\Psi_{\mathbf{H}}^{T} \mathbb{M} \Psi_{\mathbf{H}}\right)^{-1} \Psi_{\mathbf{H}}^{T}\left[\left(-\mathbb{K}+\mathbb{S}^{i}\right)\left(\mathbf{e}_{\mathbf{E}, P}^{(n+1)}+\mathbf{e}_{\mathbf{E}, V}^{(n+1)}\right)\right. \\
& \left.+\mathbb{S}^{e}\left(\mathbf{e}_{\mathbf{H}, P}^{\left(n+\frac{1}{2}\right)}+\mathbf{e}_{\mathbf{H}, V}^{\left(n+\frac{1}{2}\right)}\right)\right],
\end{aligned}\right.
$$

for $n=0,1, \cdots, N_{t}-1$ when $F_{h}^{B}=\Gamma_{a}$. Based on (17), (36), and (37), we have

$$
\mathbf{e}_{\mathbf{E}, V}^{(0)}=0, \quad \mathbf{e}_{\mathbf{H}, V}^{\left(\frac{1}{2}\right)}=0 .
$$

We first consider problem (B.1), which can be rewritten as

$$
\begin{array}{r}
{\left[\mathbb{I}-\Delta t \widehat{\Psi}\left(\widehat{\Psi}^{T} \widehat{\mathbb{M}} \widehat{\Psi}\right)^{-1} \widehat{\Psi}^{T} \mathbb{L}\right]\left[\begin{array}{c}
\mathbf{e}_{\mathbf{E}, V}^{(n+1)} \\
\mathbf{e}_{\mathbf{H}, V}^{\left(n+\frac{3}{2}\right)}
\end{array}\right]=\left[\mathbb{I}+\Delta t \widehat{\Psi}\left(\widehat{\Psi}^{T} \widehat{\mathbb{M}} \widehat{\Psi}\right)^{-1} \widehat{\Psi}^{T} \mathbb{U}\right]\left[\begin{array}{c}
\mathbf{e}_{\mathbf{E}, V}^{(n)} \\
\mathbf{e}_{\mathbf{H}, V}^{\left(n+\frac{1}{2}\right)}
\end{array}\right]} \\
+\Delta t \widehat{\Psi}\left(\widehat{\Psi}^{T} \widehat{\mathbb{M}} \widehat{\Psi}\right)^{-1} \widehat{\Psi}^{T} \mathbb{L}\left[\begin{array}{c}
\mathbf{e}_{\mathbf{E}, P}^{(n)} \\
\mathbf{e}_{\mathbf{H}, P}^{\left(n+\frac{1}{2}\right)}
\end{array}\right]+\Delta t \widehat{\Psi}\left(\widehat{\Psi}^{T} \widehat{\mathbb{M}} \widehat{\Psi}\right)^{-1} \widehat{\Psi}^{T} \mathbb{U}\left[\begin{array}{c}
\mathbf{e}_{\mathbf{E}, P}^{(n+1)} \\
\mathbf{e}_{\mathbf{H}, P}^{\left(n+\frac{3}{2}\right)}
\end{array}\right],
\end{array}
$$

and then, we have

$$
\begin{aligned}
\left\|\mathbf{e}_{\mathbf{E}, V}^{(n+1)}\right\|_{\mathbb{M}}+\left\|\mathbf{e}_{\mathbf{H}, V}^{\left(n+\frac{3}{2}\right)}\right\|_{\mathbb{M}} & \leq \beta\left(\left\|\mathbf{e}_{\mathbf{E}, V}^{(n)}\right\|_{\mathbb{M}}+\left\|\mathbf{e}_{\mathbf{H}, V}^{\left(n+\frac{1}{2}\right)}\right\|_{\mathbb{M}}\right)+\Delta t \alpha_{1}\left(\left\|\mathbf{e}_{\mathbf{E}, P}^{(n+1)}\right\|_{\mathbb{M}}+\left\|\mathbf{e}_{\mathbf{H}, P}^{\left(n+\frac{3}{2}\right)}\right\|_{\mathbb{M}}\right) \\
& +\Delta t \alpha_{2}\left(\left\|\mathbf{e}_{\mathbf{E}, P}^{(n)}\right\|_{\mathbb{M}}+\left\|\mathbf{e}_{\mathbf{H}, P}^{\left(n+\frac{1}{2}\right)}\right\|_{\mathbb{M}}\right) \\
& \leq \beta\left(\left\|\mathbf{e}_{\mathbf{E}, V}^{(n)}\right\|_{M}+\left\|\mathbf{e}_{\mathbf{H}, V}^{\left(n+\frac{1}{2}\right)}\right\|_{M}\right)+\Delta t \alpha\left(\left\|\mathbf{e}_{\mathbf{E}, P}^{(n+1)}\right\|_{M}+\left\|\mathbf{e}_{\mathbf{H}, P}^{\left(n+\frac{3}{2}\right)}\right\|_{\mathbb{M}}\right) \\
& +\Delta t \alpha\left(\left\|\mathbf{e}_{\mathbf{E}, P}^{(n)}\right\|_{\mathbb{M}}+\left\|\mathbf{e}_{\mathbf{H}, P}^{\left(n+\frac{1}{2}\right)}\right\|_{\mathbb{M})},\right.
\end{aligned}
$$

where

$$
\begin{aligned}
\beta & =\left\|\widehat{\mathbb{M}}^{\frac{1}{2}}\left[\mathbb{I}-\Delta t \widehat{\Psi}\left(\widehat{\Psi}^{T} \widehat{\mathbb{M}} \widehat{\Psi}\right)^{-1} \widehat{\Psi}^{T} \mathbb{L}\right]^{-1}\left[\mathbb{I}+\Delta t \widehat{\Psi}\left(\widehat{\Psi}^{T} \widehat{\mathbb{M}} \widehat{\Psi}\right)^{-1} \widehat{\Psi}^{T} \mathbb{U}\right] \widehat{\mathbb{M}}^{-\frac{1}{2}}\right\|_{2} \\
& =\left\|\widehat{\mathbb{M}}^{\frac{1}{2}}\left[\mathbb{I}+\Delta t \widehat{\Psi}\left(\widehat{\Psi}^{T} \widehat{\mathbb{M}} \widehat{\Psi}\right)^{-1} \widehat{\Psi}^{T} \mathbb{L}\right]\left[\mathbb{I}+\Delta t \widehat{\Psi}\left(\widehat{\Psi}^{T} \widehat{\mathbb{M}} \widehat{\Psi}\right)^{-1} \widehat{\Psi}^{T} \mathbb{U}\right] \widehat{\mathbb{M}}^{-\frac{1}{2}}\right\|_{2} \\
& >0
\end{aligned}
$$

and

$$
\alpha=\max \left\{\alpha_{1}, \alpha_{2}\right\} .
$$

By selecting

$$
\begin{aligned}
\phi_{0} & =\left\|\mathbf{e}_{\mathbf{E}, V}^{(0)}\right\|_{\mathbb{M}}+\left\|\mathbf{e}_{\mathbf{H}, V}^{\left(\frac{1}{2}\right)}\right\|_{\mathbb{M}} \\
& =0
\end{aligned}
$$

and

$$
\left\{\begin{aligned}
\phi_{n+1} & =\left\|\mathbf{e}_{\mathbf{E}, V}^{(n+1)}\right\|_{\mathbb{M}}+\left\|\mathbf{e}_{\mathbf{H}, V}^{\left(n+\frac{3}{2}\right)}\right\|_{\mathbb{M}}, \\
& \geq 0, \\
\psi_{n+1} & =\left\|\mathbf{e}_{\mathbf{E}, P}^{n+1}\right\|_{\mathbb{M}}+\left\|\mathbf{e}_{\mathbf{H}, P}^{\left(n+\frac{3}{2}\right)}\right\|_{\mathbb{M},}, \quad n=0,1, \cdots, N_{t}-1, \\
& \geq 0,
\end{aligned}\right.
$$

one can obtain the following inequality based on LEMma (1)

$$
\begin{aligned}
\Delta t \sum_{j=0}^{N_{t}}\left\|\mathbf{e}_{\mathbf{E}, V}^{(j)}\right\|_{\mathbb{M}}^{2}+\Delta t \sum_{j=0}^{N_{t}}\left\|\mathbf{e}_{\mathbf{H}, V}^{\left(j+\frac{1}{2}\right)}\right\|_{\mathbb{M}}^{2} & \leq \Delta t \sum_{i=0}^{N_{t}}\left(\left\|\mathbf{e}_{\mathbf{E}, V}^{(i)}\right\|_{\mathbb{M}}+\left\|\mathbf{e}_{\mathbf{H}, V}^{\left(i+\frac{1}{2}\right)}\right\|_{\mathbb{M}}\right)^{2} \\
& \leq 2 T_{f}\left[\alpha\left(1+\frac{1}{\beta}\right)\right]^{2}\left(\Delta t \sum_{j=0}^{N_{t}} \beta^{2 j}\right) \cdot\left[\Delta t \sum_{i=0}^{N_{t}}\left(\left\|\mathbf{e}_{\mathbf{E}, P}^{(i)}\right\|_{\mathbf{M}}+\left\|\mathbf{e}_{\mathbf{H}, P}^{\left(i+\frac{1}{2}\right)}\right\|_{\mathbb{M}}\right)^{2}\right] .
\end{aligned}
$$


The total error $\left\|\mathbf{e}_{\mathrm{ROM}}^{(j)}\right\|_{\widehat{\mathbb{M}}}^{2}\left(j=0,1, \cdots, N_{t}\right)$ satisfies

$$
\Delta t \sum_{j=0}^{N_{t}}\left\|\mathbf{e}_{\mathrm{ROM}}^{(j)}\right\|_{\mathbb{M}}^{2}=\Delta t \sum_{j=0}^{N_{t}}\left\|\mathbf{e}_{\mathbf{E}}^{(j)}\right\|_{\mathbb{M}}^{2}+\Delta t \sum_{j=0}^{N_{t}}\left\|\mathbf{e}_{\mathbf{H}}^{\left(j+\frac{1}{2}\right)}\right\|_{\mathbb{M}}^{2} .
$$

Based on (36), (37), and (B.8), we obtain

$$
\begin{aligned}
\Delta t \sum_{j=0}^{N_{t}}\left\|\mathbf{e}_{\mathrm{ROM}}^{(j)}\right\|_{\widehat{\mathbb{M}}}^{2} & =\Delta t \sum_{j=0}^{N_{t}}\left\|\mathbf{e}_{\mathbf{E}, P}^{(j)}\right\|_{\mathbb{M}}^{2}+\Delta t \sum_{j=0}^{N_{t}}\left\|\mathbf{e}_{\mathbf{E}, V}^{(j)}\right\|_{\mathbb{M}}^{2}+\Delta t \sum_{j=0}^{N_{t}}\left\|\mathbf{e}_{\mathbf{H}, P}^{\left(j+\frac{1}{2}\right)}\right\|_{\mathbb{M}}^{2}+\Delta t \sum_{j=0}^{N_{t}}\left\|\mathbf{e}_{\mathbf{H}, V}^{\left(j+\frac{1}{2}\right)}\right\|_{\mathbb{M}}^{2} \\
& \leq \Delta t \sum_{i=0}^{N_{t}}\left(\left\|\mathbf{e}_{\mathbf{E}, P}^{(i)}\right\|_{\mathbf{M}}+\left\|\mathbf{e}_{\mathbf{H}, P}^{\left(i+\frac{1}{2}\right)}\right\|_{\mathbb{M}}\right)^{2}+\left(\Delta t \sum_{j=0}^{N_{t}}\left\|\mathbf{e}_{\mathbf{E}, V}^{(j)}\right\|_{\mathbb{M}}^{2}+\Delta t \sum_{j=0}^{N_{t}}\left\|\mathbf{e}_{\mathbf{H}, V}^{\left(j+\frac{1}{2}\right)}\right\|_{\mathbb{M}}^{2}\right) .(\mathrm{B} .9)
\end{aligned}
$$

Besides, one can easily get

$$
\beta \leq 1+\gamma \Delta t \leq \mathrm{e}^{\gamma \Delta t}
$$

THEOREM 4 is proved by substituting (B.10) into (B.7) and then substituting (B.7) into (B.9). If $F_{h}^{B}=\Gamma_{a}$, one can get a similar conclusion.

[1] G. M. Oxberry, T. Kostova-Vassilevska, W. Arrighi, K. Chand, Limited-memory adaptive snapshot selection for proper orthogonal decomposition, Int. J. Numer. Meth. Engrg. 109 (2) (2017) 198-217.

[2] S. Chaturantabut, D. C. Sorensen, A state space error estimate for POD-DEIM nonlinear model reduction, SIAM J. Numer. Anal. 50 (1) (2012) 46-63.

[3] D. Amsallem, U. Hetmaniuk, Error estimates for Galerkin reduced-order models of the semi-discrete wave equation, ESAIM Math. Model. Numer. Anal. 48 (1) (2014) 135-163.

[4] L. Moya, Locally implicit discontinuous Galerkin time-domain methods for electromagnetic wave propagation in biological tissues, Ph.D. thesis, University Nice Sophia Antipolis, 2013.

[5] J. S. Hesthaven, T. Warburton, Nodal discontinuous Galerkin methods: algorithms, analysis, and applications, Springer Science \& Business Media, 2007.

[6] J. Viquerat, M. Klemm, S. Lanteri, C. Scheid, Analysis of a generalized dispersive model coupled to a DGTD method with application to nanophotonics, SIAM J. Sci. Comput. 39 (3) (2017) A831-A859.

[7] S. Dosopoulos, B. Zhao, J.-F. Lee, Non-conformal and parallel discontinuous Galerkin time domain method for maxwell's equations: EM analysis of IC packages, J. Comput. Phys. 238 (2013) 48-70.

[8] M. Lilienthal, S. M. Schnepp, T. Weiland, Non-dissipative space-time $h p$-discontinuous Galerkin method for the time-dependent maxwell equations, J. Comput. Phys. 275 (2014) 589-607.

[9] R. Léger, J. Viquerat, C. Durochat, C. Scheid, S. Lanteri, A parallel non-conforming multi-element DGTD method for the simulation of electromagnetic wave interaction with metallic nanoparticles, J. Comput. Appl. Math. 270 (2014) 330-342.

[10] L. Li, S. Lanteri, N. A. Mortensen, M. Wubs, A hybridizable discontinuous Galerkin method for solving nonlocal optical response models, Comput. Phys. Commun. 219 (2017) 99-107.

[11] L. Li, S. Lanteri, R. Perrussel, A hybridizable discontinuous Galerkin method combined to a Schwarz algorithm for the solution of 3-D time-harmonic Maxwell's equation, J. Comput. Phys. 256 (2014) $563-581$.

[12] N. C. Nguyen, J. Peraire, B. Cockburn, Hybridizable discontinuous Galerkin methods for the timeharmonic Maxwell's equations, J. Comput. Phys. 230 (19) (2011) 7151-7175.

[13] M. Rathinam, L. R. Petzold, A new look at proper orthogonal decomposition, SIAM J. Numer. Anal. 41 (5) (2003) 1893-1925. 
[14] S. Chaturantabut, D. C. Sorensen, Nonlinear model reduction via discrete empirical interpolation, SIAM J. Sci. Comput. 32 (5) (2010) 2737-2764.

[15] K. Kunisch, S. Volkwein, Galerkin proper orthogonal decomposition methods for a general equation in fluid dynamics, SIAM J. Numer. Anal. 40 (2) (2002) 492-515.

[16] S. S. Ravindran, Adaptive reduced-order controllers for a thermal flow system using proper orthogonal decomposition, SIAM J. Sci. Comput. 23 (6) (2002) 1924-1942.

[17] K. Kunisch, S. Volkwein, L. Xie, HJB-POD-based feedback design for the optimal control of evolution problems, SIAM J. Appl. Dyn. Syst. 3 (4) (2006) 701-722.

[18] M. F. de Pando, P. J. Schmid, D. Sipp, Nonlinear model-order reduction for compressible flow solvers using the discrete empirical interpolation method, J. Comput. Phys. 324 (2016) 194-209.

[19] A. Pierquin, T. Henneron, S. Clenet, S. Brisset, Model-order reduction of magnetoquasi-static problems based on POD and Arnoldi-based Krylov methods, IEEE Trans. Magn. 51 (3) (2015) 1-4.

[20] A. J. Siade, M. Putti, W. W.-G. Yeh, Snapshot selection for groundwater model reduction using proper orthogonal decomposition, Water Resour. Resear. 46 (8) (2010) 2657-2662.

[21] Z. Luo, H. Li, Y. Zhou, X. Huang, A reduced FVE formulation based on POD method and error analysis for two-dimensional viscoelastic problem, J. Math. Anal. Appl. 385 (1) (2012) 310-321.

[22] Z. Luo, F. Teng, J. Chen, A POD-based reduced-order Crank-Nicolson finite volume element extrapolating algorithm for 2-D Sobolev equations, Math. Comput. Simulat. 146 (2018) 118-133.

[23] K. Busch, J. Niegemann, M. Pototschnig, L. Tkeshelashvili, A Krylov subspace based solver for the linear and nonlinear Maxwell's equations, Phys. Status. Solidi. (b). 244 (10) (2007) 3479-3496.

[24] R.-U. Börner, O. G. Ernst, and K. Spitzer, Fast 3-D simulation of transient electromagnetic fields by model reduction in the frequency domain using Krylov subspace projection, Geophys. J. Int. 173 (3) (2008) 766-780.

[25] J. Zimmerling, L. Wei, P. Urbach, R. Remis, A Lanczos model-order reduction technique to efficiently simulate electromagnetic wave propagation in dispersive media, J. Comput. Phys. 315 (2016) 348362 .

[26] R. Mancini, S. Volkwein, An inverse scattering problem for the time-dependent Maxwell equations: nonlinear optimization and model-order reduction, Numer. Lin. Alg. Appl. 20 (4) (2013) 689-711.

[27] M. E. Kowalski, J.-M. Jin, Model-order reduction of nonlinear models of electromagnetic phasedarray hyperthermia, IEEE Trans. Biomed. Engrg. 50 (11) (2003) 1243-1254.

[28] K. Li, T.-Z. Huang, L. Li, S. Lanteri, L. Xu, B. Li, A reduced-order discontinuous Galerkin method based on POD for electromagnetic simulation, IEEE Trans. Ant. Propag. 66 (1) (2018) 242-254.

[29] Z. Luo, J. Gao, A POD reduced-order finite difference time-domain extrapolating scheme for the 2-D maxwell equations in a lossy medium, J. Math. Anal. Appl. 444 (1) (2016) 433-451.

[30] Y. Sato, and H. Igarashi, Model reduction of three-dimensional eddy current problems based on the method of snapshots, IEEE Trans. Magn. 49 (5) (2013) 1697-1700.

[31] M. Ganesh, J. S Hesthaven, B. Stamm, A reduced basis method for electromagnetic scattering by multiple particles in three dimensions, J. Comput. Phys. 231 (23) (2012) 7756-7779.

[32] Y. Chen, J. S Hesthaven, Y. Maday, J. Rodríguez, X. Zhu, Certified reduced basis method for electromagnetic scattering and radar cross section estimation, Comput. Methods Appl. Mech. Engrg. 233 (2012) 92-108.

[33] B. Peherstorfer, K. Willcox, M. Gunzburger, Survey of multifidelity methods in uncertainty propagation, inference, and optimization, SIAM Review 60 (3) (2018) 550-591. 
[34] P. Benner, S. Gugercin, K. Willcox, A survey of projection-based model reduction methods for parametric dynamical systems, SIAM Review 57 (4) (2015) 483-531.

[35] Z. Luo, Z. Xie, Y. Shang, J. Chen, A reduced finite volume element formulation and numerical simulations based on POD for parabolic problems, J. Comput. Appl. Math. 235 (8) (2011) 20982111.

[36] T. Iliescu, and Z. Wang, Are the snapshot difference quotients needed in the proper orthogonal decomposition?, SIAM J. Sci. Comput. 36 (3) (2014) A1221-A1250.

[37] D. Pasetto, M. Putti, W. W.-G. Yeh, A reduced-order model for groundwater flow equation with random hydraulic conductivity: application to Monte-Carlo methods, Water Res. Resear. 49 (6) (2013) 3215-3228.

[38] P. S. Nigro, M. Anndif, Y. Teixeira, P. M. Pimenta, P. Wriggers, An adaptive model order reduction by proper snapshot selection for nonlinear dynamical problems, Comput. Mech. 57 (4) (2016) 537554 .

[39] O. Paul-Dubois-Taine, D. Amsallem, An adaptive and efficient greedy procedure for the optimal training of parametric reduced-order models, Int. J. Numer. Methods Engrg. 102 (5) (2015) 12621292.

[40] L. Fezoui, S. Lanteri, S. Lohrengel, S. Piperno, Convergence and stability of a discontinuous Galerkin time-domain method for the 3-D heterogeneous Maxwell equations on unstructured meshes, ESAIM Math. Model. Numer. Anal. 39 (6) (2005) 1149-1176.

[41] K. Kunisch, S. Volkwein, Galerkin proper orthogonal decomposition methods for parabolic problems, Numer. Math. 90 (1) (2001) 117-148.

[42] M. Brand, Incremental singular value decomposition of uncertain data with missing values, in: European Conference on Computer Vision, Springer, 2002, pp. 707-720.

[43] A. Taflove, S. C. Hagness, Computational electrodynamics: the finite-difference time-domain method, Artech House, 2005.

[44] H. Fahs, High-order leap-frog based discontinuous Galerkin method for the time-domain maxwell equations on non-conforming simplicial meshes, Numer. Math. 2 (3) (2009) 275-300.

[45] D. Matsumoto, T. Indinger, On-the-fly algorithm for dynamic mode decomposition using incremental singular value decomposition and total least squares, ArXiv preprint - https://arxiv.org/pdf/1703.11004.pdf.

[46] T. Braconnier, M. Ferrier, J. Jouhaud, M. Montagnac, P. Sagaut, Towards an adaptive POD/SVD surrogate model for aeronautic design, Comput. Fluids. 40 (1) (2011) 195-209.

[47] G. Golub, A. Van Loan, Matrix Computations, Johns Hopkins University Press, 1996.

[48] E. Hairer, G. Wanner, S. P. Nørsett, Solving ordinary differential equations I: Nonstiff problems, Springer, 1993.

[49] G. Benderskaya, W. Ackermann, H. D. Gersem, T. Weiland, Adaptive time stepping for electromagnetic models with sinusoidal dynamics, IEEE Trans. Magn. 44 (6) (2007) 1262-1265.

[50] M. Guo, J. S. Hesthaven, Data-driven reduced order modeling for time-dependent problems, Comput. Methods Appl. Mech. Engrg. 345 (2019) 75-99.

[51] F. Vidal-Codina, N.-C. Nguyen, J. Peraire, Computing parametrized solutions for plasmonic nanogap structures, J. Comput. Phys. 366 (2018) 89-106. 